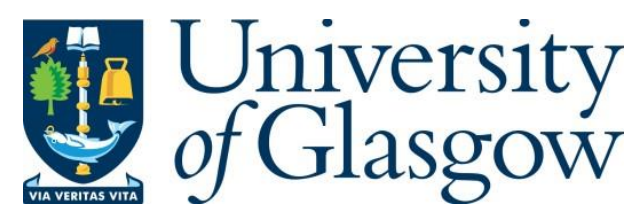

Gorgoraptis, N. et al. (2019) In vivo detection of cerebral tau pathology in long-term survivors of traumatic brain injury. Science Translational Medicine, 11(508), eaaw1993.

There may be differences between this version and the published version. You are advised to consult the publisher's version if you wish to cite from it.

http://eprints.gla.ac.uk/195004/

Deposited on: 12 September 2019

Enlighten - Research publications by members of the University of Glasgow

http://eprints.gla.ac.uk 


\title{
In vivo detection of cerebral tau pathology in long-term survivors of traumatic brain injury
}

\author{
Authors: Nikos Gorgoraptis ${ }^{1}$, Lucia M. Li ${ }^{1}$, Alex Whittington ${ }^{1,2}$, Karl A. Zimmerman ${ }^{1}$, \\ Linda M. Maclean ${ }^{3}$, Claire McLeod ${ }^{3}$, Ewan Ross ${ }^{1}$, Amanda Heslegrave ${ }^{4,5}$, Henrik \\ Zetterberg $^{4,5,6,7}$, Jan Passchier ${ }^{2}$, Paul M. Matthews ${ }^{1,8}$, Roger N. Gunn ${ }^{1,2}$, Tom M. \\ McMillan $^{3}$, David J. Sharp ${ }^{1,8 *}$
}

\begin{abstract}
Affiliations:
${ }^{1}$ Department Brain Sciences, Imperial College London, W12 0NN, UK.

${ }^{2}$ Invicro London, W12 0NN, UK.

${ }^{3}$ Institute of Health and Wellbeing, University of Glasgow, G12 0XH, UK.

${ }^{4}$ UK Dementia Research Institute, University College London, WC1N 3BG, UK.

${ }^{5}$ Department of Neurodegenerative Disease, UCL Institute of Neurology, Queen Square, London, WC1N 3BG, UK.

${ }^{6}$ Clinical Neurochemistry Laboratory, Sahlgrenska University Hospital, Mölndal, 431 80, Sweden.

${ }^{7}$ Department of Psychiatry and Neurochemistry, Institute of Neuroscience and Physiology, the Sahlgrenska Academy at the University of Gothenburg, Mölndal, 413

45, Sweden.

${ }^{8}$ UK Dementia Research Institute, W12 0NN, Imperial College London.
\end{abstract}

*To whom correspondence should be addressed: david.sharp@imperial.ac.uk 
One Sentence Summary: Positron emission tomography identified tau pathology following a single traumatic brain injury. 
Abstract: Traumatic brain injury (TBI) can trigger progressive neurodegeneration, with tau pathology seen years after a single moderate-severe TBI. Identifying this type of posttraumatic pathology in vivo might help to understand the role of tau pathology in TBI pathophysiology. We used flortaucipir positron emission tomography (PET) to investigate whether tau pathology is present many years after a single TBI in humans. We examined PET data in relation to markers of neurodegeneration in the cerebrospinal fluid (CSF), structural magnetic resonance imaging (MRI) measures and cognitive performance. Cerebral flortaucipir binding was variable, with many participants with TBI showing increases in cortical and white matter regions. At the group level, flortaucipir binding was increased in the right occipital cortex in TBI when compared to healthy controls. Flortaucipir binding was associated with increased total tau, phosphorylated tau and ubiquitin carboxy-terminal hydrolase L1 (UCH-L1) CSF concentrations, as well as with reduced fractional anisotropy and white matter tissue density in TBI. Apolipoprotein E (APOE) $\varepsilon 4$ genotype a the relationship between flortaucipir binding and time since injury, CSF $\beta$-amyloid 1-42 (A $\beta 42$ ) concentration, white matter tissue density and longitudinal mini-mental state examination (MMSE) scores in TBI. The results demonstrate that tau PET is a promising approach to investigating progressive neurodegeneration associated with tauopathy following TBI. 


\section{Introduction}

Traumatic brain injury (TBI) can lead to chronic neurodegeneration and dementia in later life $(1,2)$. Deposition of hyperphosphorylated protein tau neurofibrillary tangles is a pathological hallmark of this neurodegenerative process $(3,4)$. Since the first observations in post-mortem examinations of brain of boxers (5), the pathological features and clinical correlations of tau neurofibrillary tangle deposition in chronic traumatic encephalopathy (CTE) following repetitive TBI have been increasingly well characterized $(3,6)$. However, tau deposition also occurs following a single TBI, after which abundant and widely distributed neurofibrillary tangles have been found post-mortem in approximately one-third of TBI patients (7). As in Alzheimer's Disease (AD), all six isoforms of tau (including both 3- and 4-repeat isoforms) are observed in post-TBI tauopathy $(3,4)$. However, the distribution of tau pathology after TBI follows a pattern distinct from that observed in $\mathrm{AD}$, concentrating primarily in the depths of sulci and at points of geometric inflection in the cerebral neocortex, while the medial temporal lobe is relatively spared in early disease $(7,3)$. Although the pathogenic role of TBI as a trigger for tau aggregation is not fully understood, traumatic axonal injury appears to lead directly to tau hyperphosphorylation $(8,9)$.

Flortaucipir ([ $\left.\left.{ }^{18} \mathrm{~F}\right] \mathrm{AV}-1451,\left[{ }^{18} \mathrm{~F}\right] \mathrm{T} 807\right)$, a recently developed positron emission tomography (PET) radioligand for tau, enables the examination of tau pathology in vivo $(10,11)$. Flortaucipir demonstrated potent and specific non-displaceable binding to tau neurofibrillary tangles in post-mortem human brain tissue in AD (12-14). Flortaucipir is relatively selective for tau, with no substantial binding to $\beta$-amyloid, $\alpha$-synuclein or TDP43 in post-mortem brain tissue (12), although it also binds off-target to monoamine oxidases (15) as well as neuromelanin- and melanin-containing cells (12, 16). In vivo, flortaucipir binding is increased in $\mathrm{AD}$ patients with a regional pattern in keeping with the clinical phenotype (17-19), cognitive profile $(17,18)$ and estimated Braak \& Braak staging (20). In tau mutation carriers (MAPT gene) and in $\mathrm{AD}$, the distribution of in vivo flortaucipir binding and that of post-mortem tau pathology are strongly concordant (19, 21). Furthermore, in both typical AD and posterior cortical atrophy, flortaucipir binding follows the pattern of regional atrophy as quantified by MRI $(22,23)$. 
A recent report on 26 former National Football League players (24) and a previous single case study (25) showed increased cerebral flortaucipir binding following exposure to repetitive TBI. However the role of flortaucipir in quantifying tau pathology and its distribution in long-term survivors of a single TBI has not been studied. The relationships of tau pathology to clinical outcome and biomarkers of neurodegeneration are also unclear. Of particular interest is the relationship between tau pathology and white matter microstructural changes related to traumatic axonal injury, as these will help to clarify the pathophysiological relationship between the initial injury and progressive neurodegeneration.

Here we used flortaucipir PET to study the distribution of tau pathology in individuals at least 18 years after a single moderate-severe TBI and in healthy controls. TBI participants were primarily recruited from a patient cohort under follow-up at the University of Glasgow, who have previously been followed up longitudinally in terms of cognitive and disability outcomes $(26,27)$. We hypothesized that: i) flortaucipir binding would be increased in a proportion of participants with TBI many years after their injury; ii) flortaucipir binding would correlate with CSF and blood biomarkers of neuronal damage and neurodegeneration, including tau; iii) flortaucipir binding would be associated with the extent and distribution of diffuse axonal injury, quantified by diffusion MRI and voxel-based morphometry (VBM); and iv) flortaucipir binding would be associated with poor long-term cognitive outcomes and disability. 


\section{Results}

\section{Participant demographic and clinical characteristics}

21 participants [7 female, 14 male; median age: 49 years (range: 29 to 72)] with a history of a single moderate-severe TBI were included in the study. 19 participants with TBI were recruited from the Institute of Health and Wellbeing, Head Injury Research Group, University of Glasgow, UK, and the remaining 2 (participants P9 and P17 in Table 1) were recruited from the specialist TBI clinic at Imperial College Healthcare NHS Trust, London, UK. Participants were examined at a median time of 32 years (range: 18 to 51 years) following their injury. TBI was caused by road traffic accidents in 18 participants $(86 \%)$, assault in $2(10 \%)$ and fall from a height in 1 participant $(5 \%)$. Glasgow Outcome Scale - Extended (GOS-E) median score was 6 (range: 4 to 8) at the time of assessment. In 15 TBI participants (71\%) longitudinal clinical data were also available (27), including GOS-E and MMSE scores obtained at a median time point of 16 years (range: 9 to 19 years) after the injury and 17 years (range: 15 to 19 years) before the current clinical and imaging assessment. The demographic and clinical characteristics and APOE genotype of individual TBI participants are presented in Table 1. Focal lesions were delineated on MRI as specified in the Supplementary Materials. 19 TBI participants (90\%) had focal lesions apparent on MRI with a median volume of 1634 voxels (range: 12 to 23118 voxels). A cerebellar lesion was present on structural MRI in one participant with TBI (P7). There were no cerebellar abnormalities on structural MRI in any other participants (fig. S1).

11 healthy controls [5 female, 6 male; median age: 57 years (range: 29 to 72)] of similar educational background and estimated premorbid intelligence to the TBI participants were also examined. 8 healthy control participants were recruited from the Institute of Health and Wellbeing, Head Injury Research Group, University of Glasgow, UK, and 3 healthy controls were recruited from the NIHR Imperial Clinical Research Facility, London, UK. The demographic characteristics of the TBI and control groups are presented in Table 2.

We divided TBI participants into a good recovery and a disabled subgroup based on their Glasgow Outcome Scale - Extended scores (disabled subgroup: GOS-E $\leq 6$; good 
recovery subgroup: GOSE>6). Twelve participants [5 female, 7 male; median age: 48.5 years (range: 39 to 72)] with a median GOS-E of 5 (range: 4 to 6) were included in the disabled subgroup and 9 participants with TBI [2 female, 7 male; median age: 54 years (range: 29 to 65)] with a median GOS-E of 8 (range: 7 to 8) were included in the good recovery subgroup. Longitudinal clinical data were available for 9 participants with TBI (75\%) in the disabled and 6 participants with TBI (67\%) in the good recovery group. The demographic characteristics of these subgroups are presented in Table 2. There was no significant difference in age or years of education when each of the subgroups was compared to healthy controls (disabled TBI vs. healthy controls: age: independent t-test $\mathrm{t}=-0.91, \mathrm{P}=0.37$; education: Mann-Whitney-Wilcoxon $\mathrm{W}=60, \mathrm{P}=0.73$; good recovery $\mathrm{TBI}$ vs. healthy controls: age: $\mathrm{t}=-0.93, \mathrm{P}=0.36$; education: $\mathrm{W}=64.5, \mathrm{P}=0.26$ ). Estimated premorbid intelligence (Wechsler Test of Adult Reading, WTAR) was lower in the disabled TBI subgroup when compared to healthy controls $(\mathrm{W}=23, \mathrm{P}=0.009)$, but not significantly different between good recovery TBI and healthy controls ( $\mathrm{W}=53.5, \mathrm{P}=0.79$ ). WTAR score did not correlate with age at injury in either the disabled TBI subgroup or the TBI group overall (Spearman $\rho=-0.04, \mathrm{P}=0.89$ and $\rho=0.18, \mathrm{P}=0.43$, respectively).

\section{Neuropsychological performance}

When compared to healthy controls, participants with TBI manifested impairments on multiple cognitive domains including processing speed, executive function, motivation, inhibition, verbal and visual memory (Table 2), in a neuropsychological profile typical of TBI. On most neuropsychological measures, these differences were driven by the disabled TBI subgroup (fig. S2). The good recovery TBI subgroup had similar outcomes as healthy controls on most measures, with the exception of the Frontal Systems Behavior (FrSBe) executive and apathy scores, on which both good recovery and disabled TBI subgroups were impaired when compared to healthy controls (good recovery TBI: FrSBe-E: W=74, $\mathrm{P}=0.003$; FrSBe-A: $\mathrm{W}=63.5, \mathrm{P}=0.046$; disabled TBI: FrSBe-E: $\mathrm{W}=107.5, \mathrm{P}<0.001$; FrSBe-A: $\mathrm{W}=94, \mathrm{P}=0.005)$. The good recovery TBI subgroup performed better than the

disabled subgroup on several cognitive domains including logical reasoning, processing 
speed, executive function, motivation, inhibition, verbal memory, visual memory and mood assessment (Table 2).

As shown in Table 2, there was evidence of cognitive decline on longitudinal assessment (average -1.3 MMSE points or -0.073 MMSE points per year) in the disabled TBI subgroup whereas the good outcome TBI subgroup showed improvement of cognitive performance over time (average 1 MMSE point or 0.058 MMSE points per year), with longitudinal change being significantly different between the TBI subgroups (Table 2; $\mathrm{W}=7, \mathrm{P}=0.041)$.

\section{Flortaucipir binding in individual participants with TBI}

To assess flortaucipir binding and its distribution in the brain in individual participants with TBI, voxelwise z-scores of MNI registered flortaucipir non-displaceable binding potential $\left(\mathrm{BP}_{\mathrm{ND}}\right)$ images were derived for each patient versus the healthy control group. The resulting z-score maps describe the distribution of flortaucipir in individual participants with TBI relative to variations in binding seen in healthy subjects. As illustrated in Fig. 1, several participants with TBI had increased flortaucipir binding in a patchy cortical and subcortical distribution, involving both grey and white matter (Fig. 1: P1 to P8 and P10). In contrast, other participants with TBI had similar flortaucipir BP $\mathrm{ND}_{\mathrm{ND}}$ to that of healthy controls (Fig. 1: P17 to P22).

The number of voxels with high $\mathrm{BP}_{\mathrm{ND}}(\mathrm{z}>1.645$; corresponding to one-tailed $\mathrm{P}<0.05)$ was used to measure the spatial extent of increased flortaucipir signal in individual TBI participants (Fig. 2). Participants with TBI had a median 841 voxels above threshold (range: 0 to 15809). For comparison, voxelwise z-scores were also derived for each healthy control versus the rest of the control group. In controls, the median number of voxels above threshold was 4 (range: 0 to 253; Fig. 2).

Eight participants with TBI (38\%) had $>2000$ voxels (equivalent to $16 \mathrm{~cm}^{3}$ of brain volume) above threshold indicating spatially extensive flortaucipir signal increase, whereas a further 7 (33\%) showed increased signal of more spatially limited extent (254- 
1999 voxels, Fig. 2). The remaining 6 (29\%) had less than 253 voxels above threshold, falling within the range of the healthy control group. There was no difference in flortaucipir spatial extent when comparing between disabled and good functional outcome subgroups (Mann-Whitney-Wilcoxon $\mathrm{W}=68.5, \mathrm{P}=0.32$ ).

\section{Reduced flortaucipir binding within focal lesions in TBI}

Flortaucipir binding within focal lesions was significantly reduced when compared to non-lesioned cerebral areas (Wilcoxon signed-rank $\mathrm{V}=22, \mathrm{P}=0.002$; fig. S3). Areas of markedly reduced flortaucipir uptake (in black) in Fig. 1 correspond to focal lesions in individual TBI participants (cf fig. S1). Focal lesion size (number of voxels) did not correlate with average flortaucipir $\mathrm{BP}_{\mathrm{ND}}$ within the non-lesioned grey or white matter (Spearman $\rho=-0.37, \mathrm{P}=0.10$ and $\rho=-0.15, \mathrm{P}=0.51$, respectively; fig. S3), or with flortaucipir spatial extent (number of voxels above threshold; $\rho=-0.06, \mathrm{P}=0.78$; fig. S3).

\section{Increased flortaucipir binding in the TBI patient group vs. healthy controls}

Flortaucipir $\mathrm{BP}_{\mathrm{ND}}$ was next compared between TBI and healthy control groups, in a non-parametric voxelwise analysis, excluding lesioned areas (fig. S1) and also the striatum where off-target binding is observed with this tracer. Flortaucipir binding was significantly higher in TBI participants than in healthy controls in the right lateral occipital cortex (Fig. 3 inset; $\mathrm{P}<0.05$, adjusted for multiple comparisons). When each of the TBI subgroups was independently compared to healthy controls, significantly increased flortaucipir binding was found in both TBI subgroups, in the same right lateral occipital area (fig. S4; cf. Fig. 3; $\mathrm{P}<0.05$, adjusted for multiple comparisons). There was no significant difference in flortaucipir binding when comparing between the disabled (GOS$\mathrm{E} \leq 6$ ) and good outcome (GOS-E>6) TBI subgroups (fig. S4). There were no areas of significantly decreased binding in the TBI group or subgroups compared to healthy controls (fig. S4; all P>0.05).

Additionally, we examined flortaucipir $\mathrm{BP}_{\mathrm{ND}}$ within a medial temporal lobe region of interest (ROI) including the entorhinal, perirhinal and parahippocampal cortex and the 
hippocampus, a region typically affected by tau accumulation in Alzheimer's disease. There was no significant difference in flortaucipir binding within this ROI between the TBI group, or either of the disabled or good recovery TBI subgroups, and healthy controls (fig. S5 Mann-Whitney-Wilcoxon; all TBI vs. healthy controls: $\mathrm{W}=108, \mathrm{P}=0.78$; disabled TBI vs. healthy controls: $\mathrm{W}=57, \mathrm{p}$-value $=0.61$; good recovery TBI vs. healthy controls: $\mathrm{W}=51, \mathrm{P}=0.94)$.

\section{Correlations between flortaucipir and CSF T-tau, P-tau and UCH-L1 in TBI}

Next, CSF and plasma biomarkers of neurodegeneration and neuronal damage, including total tau (T-tau), tau phosphorylated at amino acid 181 (P-tau), ubiquitin carboxy-terminal hydrolase L1 (UCH-L1), $\beta$-amyloid 1-42 (A $\beta 42)$, neurofilament light (NFL), glial fibrillary acidic protein (GFAP) and protein S100 were assessed in TBI and healthy controls and their relationship with flortaucipir binding was explored. CSF data were available from 19 participants with TBI and 10 healthy controls.

In TBI, CSF T-tau was significantly correlated with flortaucipir $\mathrm{BP}_{\mathrm{ND}}$ in the cerebral cortical grey matter (Spearman $\rho=0.53, \mathrm{P}=0.022$; Fig. $4 \mathrm{~A}$ ), but not in the white matter ( $\rho=0.44, \mathrm{P}=0.059$; fig. S6). Conversely, CSF P-tau correlated with flortaucipir $\mathrm{BP}_{\mathrm{ND}}$ in the cerebral white matter $(\rho=0.52, \mathrm{P}=0.024$; Fig. 4B), but not in the grey matter $(\rho=0.32$, $\mathrm{P}=0.18$; fig. S6). There was no correlation between CSF T-tau or P-tau and flortaucipir spatial extent (T-tau: $\rho=0.21, \mathrm{P}=0.38$; P-tau: $\rho=0.29, \mathrm{P}=0.24$; fig. S6). CSF tau in healthy controls did not correlate with flortaucipir binding in the cerebral grey matter (T-tau: $\rho=0.15, \mathrm{P}=0.68$; P-tau: $\rho=-0.21, \mathrm{P}=0.56$; fig. S6) or white matter (T-tau: $\rho=-0.09, \mathrm{P}=0.81$; P-tau: $\rho=0, \mathrm{P}=1$; fig. S6). Plasma T-tau concentration did not correlate with flortaucipir binding in either TBI or healthy controls (fig. S6). There were no differences in T-tau or P-tau concentration in CSF or plasma between TBI and healthy control groups (CSF Ttau: $\mathrm{W}=93, \mathrm{P}=0.95$; CSF P-tau: $\mathrm{W}=67, \mathrm{P}=0.21$; plasma T-tau: $\mathrm{W}=118, \mathrm{P}=0.76$; fig. $\mathrm{S} 6$ ).

UCH-L1 in the CSF in TBI participants also positively correlated with flortaucipir $\mathrm{BP}_{\mathrm{ND}}$ in the grey matter (Spearman $\rho=0.52, \mathrm{P}=0.023$; Fig. 4C), but not in the white matter $(\rho=0.42, \mathrm{P}=0.073$; fig. S7). There was no association between plasma UCH-L1 and 
flortaucipir binding in TBI (fig. S7). In healthy controls, there were no associations between flortaucipir binding and UCH-L1 in the CSF or plasma (fig. S7). There was no difference between TBI and control groups in UCH-L1 concentration (fig. S7). There were also no associations between flortaucipir $\mathrm{BP}_{\mathrm{ND}}$ and $\mathrm{A} \beta 42$, NFL, GFAP or S100 in TBI or healthy controls and no difference between TBI and control groups in the CSF or plasma (fig. S7).

\section{Flortaucipir spatial extent and white matter damage in TBI}

Next, we investigated the relationship between flortaucipir binding and structural brain injury produced by TBI (28). White matter integrity measured by fractional anisotropy (FA) was significantly reduced in TBI when compared to healthy controls (Mann-Whitney-Wilcoxon $\mathrm{W}=44, \mathrm{P}=0.004$; Fig. 5A), in keeping with the presence of traumatic axonal injury (TAI) in TBI. As expected in TBI (29), reduced FA correlated with worse performance on multiple cognitive measures (table S1). Tissue density measured by voxel-based morphometry (VBM) was also significantly reduced in the non-lesioned white matter in TBI (W=61, $\mathrm{P}=0.031$; Fig. 5B). Tissue density in the non-lesioned grey matter was not significantly different between TBI and healthy control groups $(\mathrm{W}=113, \mathrm{P}=0.94$; Fig. 5C).

The spatial extent of flortaucipir binding (number of voxels with $\mathrm{BP}_{\mathrm{ND}} \mathrm{Z}>1.645$ ) correlated with the degree of white matter damage seen in TBI participants. The number of voxels showing increased flortaucipir binding was negatively correlated with both average FA (Spearman $\rho=-0.48, \mathrm{P}=0.027$; Fig. 5D) and average white matter density ( $\rho=-$ $0.46, \mathrm{P}=0.037$; Fig. 5E), in keeping with a relationship between cerebral tau pathology with microstructural white matter disruption. There was no significant correlation between flortaucipir spatial extent and grey matter tissue density ( $\rho=-0.31, \mathrm{P}=0.17$; Fig. 5F).

\section{White matter damage in areas of increased flortaucipir binding}

We next examined whether greater white matter pathology was seen in areas of increased flortaucipir binding. As the localisation of increased tau varies from patient to 
patient, we defined areas of high and normal flortaucipir signal $\left(\mathrm{BP}_{\mathrm{ND}} \mathrm{z}>\right.$ or $\left.<1.645\right)$ in each patient. Eight TBI participants who did not show high flortaucipir signal within the white matter were excluded from these analyses. Significantly lower FA (Fig. 6A-B; Wilcoxon signed-rank $\mathrm{V}=19, \mathrm{P}=0.018$ ) and white matter density (Fig. 6C-D; V=17, $\mathrm{P}=0.025$ ) was seen in areas of high flortaucipir binding compared to areas where binding was not increased, demonstrating relatively high flortaucipir binding in areas of posttraumatic white matter damage. The corresponding VBM analysis for grey matter density did not reveal differences in grey matter density between areas of high and those of normal flortaucipir binding ( $\mathrm{V}=97, \mathrm{P}=0.95$; fig. S8). There was considerable between-subject variability in these effects (Fig. 6B and D, fig. S8B).

\section{Cortical flortaucipir binding and white matter microstructural damage}

Next, we examined whether flortaucipir $\mathrm{BP}_{\mathrm{ND}}$ within the right lateral occipital cortical area where flortaucipir signal was increased in TBI vs. controls (Fig. 3) was associated with white matter tract FA. Increased right lateral occipital flortaucipir binding was associated with reduced FA in remote white matter regions, including association, commissural and projection tracts (Fig. 7A, B and table S2). Correlations were observed in the genu and body of the corpus callosum, as well as in several association tracts within the ipsilateral (right) hemisphere, including the cingulum bundle, inferior longitudinal fasciculus, uncinate fasciculus and anterior thalamic radiation (Fig. 7B and table S2), but not in the contralateral hemisphere (fig. S9 and table S2). Higher cortical flortaucipir BP $\mathrm{ND}_{\mathrm{N}}$ was associated with reduced tissue density in remote white matter regions including the corpus callosum and right prefrontal white matter (Fig. 7C). The same analysis for grey matter density did not show an association between flortaucipir $\mathrm{BP}_{\mathrm{ND}}$ within the lateral occipital cluster and cerebral cortical grey matter density $(\mathrm{P}>0.05$ corrected for multiple comparisons).

\section{Flortaucipir binding and clinical measures in TBI}

There were no correlations of average flortaucipir $\mathrm{BP}_{\mathrm{ND}}$ in the cerebral grey or white matter or the spatial extent of high flortaucipir signal in TBI participants (number of 
voxels above $\mathrm{BP}_{\mathrm{ND}} \mathrm{z}>1.645$ ) with age, time since injury, disability (GOS-E) or any neuropsychological measures in the TBI participants (all $\mathrm{P}>0.05$, false discovery rateadjusted, table S3).

APOE $\& 4$ genotype interactions with flortaucipir binding and time since injury, CSF Aß42, white matter density and MMSE scores in TBI

There were no overall differences between participants with at least one APOE $\varepsilon 4$ allele and those without an $A P O E \& 4$ allele in either cerebral flortaucipir BP $\mathrm{ND}_{\mathrm{N}}$ or in flortaucipir spatial extent in the TBI group (Mann-Whitney-Wilcoxon: $\mathrm{W}=41, \mathrm{P}=0.46$; $\mathrm{W}=44, \mathrm{P}=0.60$, respectively; fig. $\mathrm{S} 10)$.

In further exploratory analyses, we examined whether $A P O E$ carrier status (presence of at least one $\varepsilon 4$ allele) influenced the relationship between flortaucipir measures (average cerebral $\mathrm{BP}_{\mathrm{ND}}$ and spatial extent) and age, time since injury, CSF biomarkers, white matter MRI measures, and neuropsychological performance in TBI. Interactions between $A P O E$ status and each of the above variables on flortaucipir measures were examined using linear regression in the TBI group. Interaction plots are presented in fig. S11.

There was an interaction between $A P O E$ genotype and time since injury on cerebral flortaucipir $\mathrm{BP}_{\mathrm{ND}}\left(F_{(1,17)}=15.11, \mathrm{P}=0.001\right)$, but not on flortaucipir spatial extent. Flortaucipir $\mathrm{BP}_{\mathrm{ND}}$ increased with time elapsed since the TBI in $A P O E \varepsilon 4$ carriers, but not in $\varepsilon 4$ non-carriers (fig. S11). Age did not interact with $A P O E$ genotype on average cerebral flortaucipir BP $\mathrm{ND}$ or on flortaucipir spatial extent (fig. S11).

There was an interaction between $A P O E$ genotype and CSF A $\beta 42$ on flortaucipir $\operatorname{BP}_{\mathrm{ND}}\left(F_{(1,15)}=6.15, \mathrm{P}=0.026\right)$, but not on flortaucipir spatial extent in TBI (fig. S11). Higher cerebral flortaucipir binding was associated with lower A $\beta 42$ concentration in CSF in $A P O E \varepsilon 4$ carriers, but not in $\varepsilon 4$ non-carriers (fig. S11). There were no interactions on flortaucipir measures between $A P O E \& 4$ carrier status and T-tau, P-tau, UCH-L1, NFL, GFAP, or S100 CSF concentrations (fig. S11). 
The effect of white matter tissue density (derived from voxel based morphometry) on flortaucipir spatial extent was influenced by $A P O E$ genotype $\left(\mathrm{F}_{(1,17)}=6.90, \mathrm{P}=0.018\right)$, with $\varepsilon 4$ non-carriers showing increased flortaucipir binding with reducing white matter tissue density, which was not observed in $A P O E \& 4$ carriers (fig. S11). There were no interactions between white matter tissue density and $A P O E$ genotype on average cerebral flortaucipir $\mathrm{BP}_{\mathrm{ND}}$ and no interactions between grey matter tissue density or fractional anisotropy and APOE genotype on flortaucipir measures (fig. S11).

$A P O E$ genotype influenced the relationship of longitudinal Mini-Mental State Exam (MMSE) scores and flortaucipir binding, with decreasing MMSE scores over time associated with higher cerebral flortaucipir $\mathrm{BP}_{\mathrm{ND}}$ in $\varepsilon 4$ non-carriers, but not in $\varepsilon 4$ carriers $\left(F_{(1,10)}=40.76, \mathrm{P}<0.0001\right.$, fig. S11). Current MMSE scores also interacted with APOE \&4 carrier status on flortaucipir spatial extent, with current MMSE scores being lower with increasing spatial extent in $\varepsilon 4$ non-carriers, but not in $\varepsilon 4$ carriers $\left(F_{(1,17)}=6.14, \mathrm{P}=0.024\right.$, fig. S11.

\section{Similar off-target binding in TBI and healthy controls}

In keeping with the known off-target binding of flortaucipir to neuromelanin (12), flortaucipir BP $\mathrm{ND}$ was increased in the striatum in both TBI participants and in healthy controls when compared to the rest of the cerebral white and grey matter, excluding focal lesions (Wilcoxon signed-rank TBI: $\mathrm{V}=214, \mathrm{P}=0.0002$; controls: $\mathrm{V}=66, \mathrm{P}=0.001$; fig. $\mathrm{S} 12)$. However, flortaucipir $\mathrm{BP}_{\mathrm{ND}}$ in the striatum and in the choroid plexus (16) were not different between TBI participants and healthy controls (fig. S12). 


\section{Discussion}

Hyperphosphorylated tau deposition is a key pathological feature of neurodegeneration triggered by traumatic brain injury (TBI) $(3,4)$. Patients are at increased risk of Alzheimer's disease (AD) and chronic traumatic encephalopathy (CTE) after TBI, both characterized by the accumulation of tau pathology $(1,30,31)$. Positron emission tomography (PET) ligands have recently been developed that specifically bind hyperphosphorylated tau, enabling the detection of tau pathology in vivo (32). We used flortaucipir PET to investigate tau pathology in a well-characterized cohort of individuals who were examined many years after a single moderate-severe $\operatorname{TBI}(26,27)$. As predicted, there was considerable variability in the extent of flortaucipir binding across TBI participants. Broadly, a third of TBI participants showed extensive increases in cerebral flortaucipir binding, a third showed more limited increases and a third showed no abnormality. The proportion of individuals with increased flortaucipir binding is much higher than would be expected in the general population in this age group (33). At the group level, TBI participants showed elevated flortaucipir binding when compared to ageand education-matched healthy controls.

Flortaucipir binds to neurofibrillary tangles containing abnormal tau in postmortem human brain tissue in AD (12-14). A lack of specificity has been a critical issue with previous PET tau ligands (34), but flortaucipir is more selective and does not show substantial $\beta$-amyloid, $\alpha$-synuclein or TDP-43 binding in post-mortem brain tissue (12). However, off-target binding to monoamine oxidases and neuromelanin- and melanincontaining cells is a known limitation $(12,15,16)$. Flortaucipir has been validated for the study of tau pathology in $\mathrm{AD}$ patients, showing binding that correlates with clinical phenotype (17-19), cognitive profile $(17,18)$ and estimated Braak \& Braak staging (20). Tau pathology seen after TBI appears to be similar to AD, with all six isoforms of tau (including both 3- and 4-repeat isoforms) observed $(3,4)$. As a result, flortaucipir is expected to bind tau pathology seen after TBI in a similar way to $\mathrm{AD}$, albeit with a distinct spatial pattern (3).

The pattern of flortaucipir binding potentially provides diagnostic information about the type of post-traumatic neurodegeneration. Tau pathology seen after TBI often 
has a distribution distinct from AD. In cases of CTE, tau pathology is concentrated in depths of cortical sulci $(3,6)$ and can be widely distributed in cortical areas following single TBI (7). In our study some patients showed extensive flortaucipir binding in cortical areas as well as in parts of the white matter. This spatial pattern would be atypical for AD (35) but is more in keeping with $\operatorname{CTE}(3,4)$. It is also compatible with the spatially extensive tau neurofibrillary tangle pathology seen in approximately one-third of chronic TBI patients (7).

The relationship between tau pathology and flortaucipir binding is supported by our CSF results. CSF tau is raised in incipient AD (36) and other tauopathies (37) and provides a diagnostic biomarker for AD (38). CSF tau increases in the days after TBI, generally falling to normal concentration 8-12 weeks after the injury $(39,40)$. To our knowledge, our study is the first to examine CSF tau several years after moderate-severe TBI. CSF T-tau and P-tau were not increased across the TBI group, which might be due to the high variability within the TBI group. CSF T-tau and P-tau concentrations correlated positively with flortaucipir binding in the cerebral cortex and white matter, respectively, supporting the conclusion that increased flortaucipir binding is indicative of the presence of tau pathology. We also observed a positive correlation between CSF UCH-L1 concentration and cortical flortaucipir uptake. In the acute phase, UCH-L1 may indicate the presence of neuronal injury following TBI (41). It is abundant in cerebral neurons and is an important component of the ubiquitin-proteasome system, (42). Dysfunction of UCHL1 is implicated in a number of neurodegenerative diseases $(43,44)$ and UCH-L1 is found within misfolded protein aggregates, including neurofibrillary tangles (45). CSF UCH-L1 is elevated in patients with Alzheimer disease (46) and our results suggest that the link between TBI and dysfunction in the ubiquitin-proteasome system warrants further investigation.

We also showed a relationship between flortaucipir binding and traumatic axonal injury (TAI). This is in keeping with a causative role for TAI in the pathophysiology of post-traumatic tau pathology. Mechanical forces exerted at the time of head injury are thought to disrupt axonal organization producing damage to microtubule structure and associated axonal tau (4). This damage may lead to hyperphosphorylation of tau, misfolding, and neurofibrillary tangle formation, which eventually causes 
neurodegeneration $(8,9,47)$. Mechanical forces are maximal in points of geometric inflection such as the base of cortical sulci (48), where tau pathology is seen in CTE.

In $\mathrm{AD}$, increased flortaucipir binding is greater in cortical regions that show atrophy $(22,23)$. We observed a different relationship following TBI. Flortaucipir binding was increased in white matter areas that also showed reduced fractional anisotropy and atrophy. This suggests that tau pathology was more marked in areas of TAI. In addition, cortical flortaucipir binding also correlated with the extent of TAI within the white matter. For example, flortaucipir binding in the right lateral occipital cortex, the most consistent area of increase, was correlated with reduced fractional anisotropy in a tract directly connected to that cortical area, the inferior longitudinal fasciculus. This relationship might be explained by slow Wallerian degeneration that follows the initial injury and leads to the accumulation of tau pathology in connected cortical regions (49). Alternatively, prion-like spread of tau-pathology might over time result in the cortical accumulation of taupathology in regions that are connected to white matter regions initially damaged by TAI $(50,51)$. We have previously reported a similar relationship between cortical amyloid pathology measured using $\left[{ }^{11} \mathrm{C}\right]-\mathrm{PiB}$ PET and the degree of TAI in connected tracts $(52)$, suggesting that structural connectivity may influence the neuroanatomical distribution of post-traumatic tau and amyloid pathology in similar ways.

A recent study on former National Football League (NFL) players, exposed to repetitive TBI and manifesting cognitive, mood and behavioral symptoms, also found raised cerebral flortaucipir binding, which increased with years of exposure to repetitive TBI (24). With our results, this supports the hypothesis that the risk for neurodegeneration from TBI may be dose-dependent (1), produced either by a single severe TBI or repeated exposure to more minor TBI.

Genetic factors may partially explain the variability in the neurodegenerative trajectory following $\mathrm{TBI}(53,54)$. APOE $\varepsilon 4$, the strongest risk factor for sporadic $\mathrm{AD}$, is associated with increased $\beta$-amyloid and tau pathology (55), and it is also the strongest genetic predictor of adverse outcome after TBI (56). APOE $\varepsilon 4$ may act synergistically with TBI to elevate the risk of neurodegeneration $(30,57)$. Therefore, in exploratory analyses, we examined how $A P O E \& 4$ carrier status might influence flortaucipir binding. Flortaucipir 
binding increased with time since TBI in $A P O E \varepsilon 4$ carriers and was associated with lower A $\beta 42$ concentrations in the CSF (indicating higher $A \beta 42$ concentrations in the brain) in $\varepsilon 4$ carriers. This supports an influence of $A P O E \varepsilon 4$ on the risk of neurodegeneration after TBI. In keeping with these findings, $A \beta$ plaque pathology following TBI is more prevalent in individuals with at least one $A P O E \varepsilon 4$ allele (57). Results from animal models also support the association between $A P O E \varepsilon 4$ genotype and A $\beta$ pathology after TBI (58).

Our study has a number of limitations. The relatively small sample size may have limited our ability to detect associations between flortaucipir binding and clinical outcomes. Hence, the absence of a clinical effect should be interpreted with caution, as our study is unlikely to have been adequately powered for these outcomes. In AD, flortaucipir uptake was associated with poorer performance in various cognitive domains in regionally specific patterns (17). However, the situation is likely to be more complex following TBI as the influence of the initial injury on cognitive state will confound progressive neurodegenerative effects, resulting in the need for larger sample sizes. Estimated premorbid intelligence was lower in the disabled TBI subgroup when compared to healthy controls. However, vocabulary development may be adversely affected by TBI during development (59). Hence, it is possible that performance on WTAR was directly reduced by the TBI, especially in individuals injured during childhood. Flortaucipir binds off-target to monoamine oxidases and neuromelanin, which is particularly apparent in the striatum and choroid plexus $(12,13,15,16)$. This off-target flortaucipir binding may have limited our ability to detect underlying tau pathology in these regions. In addition, a contribution from non-tau pathology cannot be excluded with certainty. Flortaucipir binding increases have been reported in semantic variant primary progressive aphasia, where the underlying pathology is TDP-43 (60), although flortaucipir binding to TDP-43 has not been demonstrated post-mortem (12). Off-target binding has also been reported in areas of acute parenchymal and subarachnoid haemorrhage, but not in areas showing superficial siderosis due to chronic haemorrhage (12). This is unlikely to be a major confound in our study as there were no acute haemorrhagic lesions in our participants and binding was reduced within focal lesions. Flortaucipir signal is reduced at the site of focal lesions. Therefore, the presence of a focal lesion in one or more TBI participants within a given brain area would be expected to reduce our sensitivity in detecting flortaucipir group effects within 
that area. To minimize the impact of lesions in our analysis we took into account the location of focal lesions as regressors in voxelwise group comparisons. Finally, brain atrophy can impact on PET results, but we employed stringent measures to minimise the effects of atrophy during pre-processing. Moreover, the relationship between increased flortaucipir binding and reduced white matter tissue density is unlikely to be artifactual, as non-specific flortaucipir binding is expected to be reduced within areas of reduced tissue density.

In summary, we show that flortaucipir binding is increased many years after a single moderate-severe TBI. Increased binding was associated with CSF indicators of neurodegeneration, including T-tau and P-tau, as well as the presence of TAI that may provide the initial trigger to its accumulation. The ability to detect tau pathology in vivo following TBI has major potential implications for diagnosis and prognostication of clinical outcomes after TBI. It is also likely to assist in patient selection and stratification for future treatment trials targeting tau. 


\section{Materials and Methods}

\section{Study design}

This study was designed to assess whether flortaucipir PET provides evidence of tau pathology in individuals several years after a single moderate-severe TBI when compared to healthy controls. We also examined the relationship of flortaucipir signal with CSF and blood biomarkers, MRI measures of axonal injury and cognitive and disability outcomes. Imaging assessment and biological sampling was cross-sectional and longitudinal cognitive and disability measures were available in a subset of individuals with TBI. The sample size was calculated to provide $80 \%$ statistical power with a type 1 error rate of 0.05 to detect an increase in flortaucipir binding in TBI compared to controls, based on effect sizes extrapolated from previous flortaucipir data on $\mathrm{AD}$ (11), and from post-mortem data showing tau pathology in about a third of individuals following moderate-severe TBI (7). Investigators performing the PET modeling, MRI processing and lesion delineation were blinded as to clinical outcomes and biomarker data. Randomization was not applicable in this observational study.

TBI participants were primarily recruited from two TBI cohorts under follow-up at the Institute of Health and Wellbeing, Head Injury Research Group, University of Glasgow, UK. The original cohorts included all individuals acutely admitted with TBI to the Southern General Hospital, Glasgow, between 1968 and 1985 (27) and between 1996 and 1999 (26). These individuals have been followed up longitudinally in terms of cognitive, wellbeing and disability outcomes $(26,27)$. Members of these follow-up cohorts fulfilling the inclusion criteria for the current study were invited to participate with the assent of their general practitioner (primary care physician). Additional TBI participants fulfilling the inclusion criteria were recruited from the specialist multidisciplinary TBI clinic at Imperial College Healthcare NHS Trust, London, UK. Individuals were recruited into the TBI group with the following inclusion criteria: i) a history of a single moderatesevere TBI (according to Mayo classification (61)), ii) age over 18 years, iii) capacity to provide written informed consent, iv) no prior neurological or psychiatric illness, v) no contraindication to PET or prior radiation exposure which when combined with the dose from the present study would exceed $10 \mathrm{mSv}$ in addition to the natural background 
radiation in the previous 3 years, vi) no contraindication to MRI, vii) no medication use or allergies which may compromise participant safety or interfere with study procedures. TBI participants were divided into two subgroups based on functional outcome. Poor functional outcome was defined as a GOS-E score of 6 or less (moderate-severe disability) at the time of assessment and those with GOS-E greater than 6 as good outcome. Healthy volunteers of similar age and socioeconomic background as the TBI participants were also recruited. The study was approved by the Westminster Research Ethics Committee and the Administration of Radioactive Substances Advisory Committee. All participants gave written informed consent.

\section{Procedures}

All participants were administered an IV bolus of flortaucipir (also known as $\left[{ }^{18} \mathrm{~F}\right] \mathrm{AV}-1451$ and $\left.\left[{ }^{18} \mathrm{~F}\right] \mathrm{T} 807\right)$, average dose $250 \mathrm{MBq}$ and dynamic PET scans were acquired over 90 minutes. Flortaucipir was supplied by Avid Radiopharmaceuticals, a wholly owned subsidiary of Eli Lilly and Company and PET image acquisition was carried out at Imanova Centre for Imaging Sciences (currently Invicro London). Flortaucipir PET data was analyzed with the Molecular Imaging and Kinetic Analysis Toolbox (MIAKAT www.miakat.org) using the simplified reference tissue model (SRTM) $(62,63)$ with cerebellar grey matter as the reference tissue, based on post-mortem studies in chronic traumatic encephalopathy which demonstrated relative sparing from tau pathology in that region (3, 64). All participants also underwent structural 3T MRI, including volumetric T1 and diffusion tensor imaging (DTI). Details on PET and MRI acquisition and analyses are provided in Supplementary Materials.

TBI and control participants took part in neuropsychological assessment and had venous blood and cerebrospinal fluid (CSF) sampling. Clinical assessment, blood and CSF sample acquisition were carried out at the NIHR/Welcome Trust Imperial Clinical Research Facility. Details on neuropsychological assessments and biological sample analyses are provided in Supplementary Materials. 


\section{Statistical analyses}

Statistical analyses were carried out using $\mathrm{R}$ statistical software (www.rproject.org) (65) unless specified otherwise.

Voxelwise differences in $\mathrm{BP}_{\mathrm{ND}}$ between $\mathrm{TBI}$ participants and healthy controls, between each of the disabled TBI and good recovery TBI subgroups and healthy controls as well as between these subgroups were assessed using permutation tests in FSL (66). The same procedure was used to assess voxelwise differences between TBI participants and healthy controls in: i) VBM-derived white matter density ii) grey matter density and iii) diffusion MRI-derived skeletonized fractional anisotropy (FA). Voxels corresponding to structural lesions were excluded from the analyses by using subjects' lesion maps as individual voxelwise repressors. All cerebral grey and white matter voxels were included except those corresponding to the striatum, where there is known off-target binding (12). 1000 permutations were used and results were cluster-corrected for multiple comparisons using threshold-free cluster enhancement (TFCE) and a family-wise error rate of 0.05. Localisation of voxel clusters was reported based on the Harvard-Oxford probabilistic atlas (67) and the JHU DTI-based white matter atlas (68).

To investigate whether white matter tau pathology shared common localisation with traumatic axonal injury and / or white matter atrophy in TBI, we compared skeletonized FA and, separately, average white matter density (z-scores) within areas of high flortaucipir signal $\left(\mathrm{BP}_{\mathrm{ND}} \mathrm{Z}>1.645\right)$ versus white matter areas where binding was not increased $\left(\mathrm{BP}_{\mathrm{ND}} \mathrm{z}<1.645\right)$ using (paired) Wilcoxon signed-rank tests. Any voxels corresponding to focal lesions were excluded. Only TBI participants with 15 or more white matter voxels with increased flortaucipir binding within the regions of interest were included.

To examine the localisation of white matter microstructural damage predicted by increased flortaucipir cortical signal in TBI participants, standardized $\mathrm{BP}_{\mathrm{ND}}$ within the right parietal cortical area of flortaucipir increase in TBI was used as a regressor in a voxelwise GLM of skeletonised FA, and separately, in a voxelwise GLM of white matter density. Statistical significance was tested using permutation tests in FSL (66). As previously, voxels corresponding to lesions were excluded for each individual, 1000 
permutations were used and results were obtained using TFCE, corrected for multiple comparisons using a family-wise error rate of 0.05. Localisation of voxel clusters was reported based on the JHU DTI-based white matter atlas (68). Post-hoc correlations were examined using Spearman's rank correlation between standardized $\mathrm{BP}_{\mathrm{ND}}$ within the right parietal cortical area of flortaucipir increase in TBI participants and skeletonized FA in the following tracts: right and left superior longitudinal, inferior longitudinal, inferior frontooccipital fasciculi and cingulum bundles, corpus callosum (genu, body and splenium) as well as in the right and left corticospinal tracts as a control.

Correlations between clinical/neuropsychological measures and PET markers in TBI participants were examined using Spearman's rank correlation with false discovery rate (FDR) correction for multiple comparisons. Interactions between APOE genotype (presence of at least one $\varepsilon 4$ allele) and age, time since injury, CSF biomarkers, white matter MRI measures, and neuropsychological performance on cerebral flortaucipir $\mathrm{BP}_{\mathrm{ND}}$ and flortaucipir spatial extent were examined in the TBI group using linear regression in each case to assess the statistical significance of the interaction term, and interaction plots to determine the direction of the effect in $\varepsilon 4$ carriers vs non-carriers. Assumptions of multiple linear regression analysis were tested as follows: analysis of standard residuals was carried out to identify outliers; variance inflation factor was used to test for multicollinearity; Durbin-Watson test was conducted to test for independence of errors; approximate normal distribution of errors, homoscedacity and linearity were assessed by plotting regression standardized residuals.

Additional Materials and Methods are provided in the Supplementary Materials. 


\section{Supplementary Materials}

Materials and Methods

Fig. S1. Focal lesions in TBI participants.

Fig. S2: Individual data points as well as means and standard deviations for demographic and neuropsychological results in the healthy control group (HC), disabled TBI subgroup (TBI-D) and good recovery TBI subgroup (TBI-G).

Fig. S3: Flortaucipir binding and focal lesions. A) Reduced flortaucipir $\mathrm{BP}_{\mathrm{ND}}$ within focal lesions compared to non-lesioned cerebral areas. No correlation between lesion size (number of lesioned voxels) and B) grey matter (GM), or C) white matter (WM) average flortaucipir $\mathrm{BP}_{\mathrm{ND}}$, or D) flortaucipir spatial extent.

Fig. S4: Voxelwise comparisons between subgroups in flortaucipir binding.

Fig. S5: Flortaucipir medial temporal lobe $\mathrm{BP}_{\mathrm{ND}}$ in the in the healthy control $(\mathrm{HC})$, disabled TBI (TBI-D) and good recovery TBI (TBI-G) groups.

Fig. S6: Relationships of CSF T-tau and P-tau and plasma T-tau concentrations with flortaucipir (FTP) grey matter (GM) $\mathrm{BP}_{\mathrm{ND}}$, white matter (WM) $\mathrm{BP}_{\mathrm{ND}}$ and spatial extent in TBI and healthy controls.

Fig. S7: Relationships of CSF and plasma UCH-L1, NFL, GFAP, S100 and CSF A $\beta 42$ with cerebral grey matter $(\mathrm{GM})$ and white matter (WM) flortaucipir $\mathrm{BP}_{\mathrm{ND}}$ in $\mathrm{TBI}$ and healthy controls.

Table S1. Correlations between neuropsychological measures and average white matter tract fractional anisotropy in TBI.

Fig. S8: Grey matter density within areas of increased flortaucipir $\mathrm{BP}_{\mathrm{ND}}$ in $\mathrm{TBI}$.

Fig. S9: Flortaucipir right parietal cluster $(\mathrm{TBI}>\mathrm{Control}) \mathrm{BP}_{\mathrm{ND}}$ (z-score compared to healthy controls) and fractional anisotropy (FA) in white matter tracts in TBI. 
Table S2. Correlations between Flortaucipir right parietal cluster (TBI>Control) BPND (zscore compared to healthy controls) and fractional anisotropy (FA) in white matter tracts in TBI participants.

Table S3. Correlations between clinical measures and each of: flortaucipir right parietal cluster (TBI>Control) $\mathrm{BP}_{\mathrm{ND}}$, whole cerebral cortex $\mathrm{BP}_{\mathrm{ND}}$ (z-score compared to the healthy control group), white matter $\mathrm{BP}_{\mathrm{ND}}$ (z-score compared to the healthy control group) and spatial extent ( $\mathrm{N}$ of voxels with $\mathrm{BP}_{\mathrm{ND}} \mathrm{z}$-score>1.645 of healthy controls) in $\mathrm{TBI}$ participants.

Fig. S10: No difference in flortaucipir BPND (z-score compared to healthy controls) or spatial extent (number of voxels above threshold) in TBI when comparing individuals with at least one $\operatorname{APOE} \varepsilon 4$ allele and those not carrying an $\varepsilon 4$ allele.

Fig. S11: Interactions between $A P O E$ genotype and each of time since injury, age, CSF Ttau, P- tau, A 342 , NFL, GFAP, S100 concentrations, white matter (WM) and grey matter (GM) densities and MMSE scores (longitudinal change and current) on Flortaucipir BP $\mathrm{ND}_{\mathrm{N}}$ (z-score compared to controls and spatial extent ( $\mathrm{N}$ voxels above threshold) in TBI.

Fig. S12: Off-target flortaucipir binding in the striatum and choroid plexus compared to other cerebral areas in healthy controls and TBI.

Fig. S13. Flortaucipir reference region time activity curves (TAC). 


\section{References and Notes}

1. D. H. Smith, V. E. Johnson, W. Stewart, Chronic neuropathologies of single and repetitive TBI: substrates of dementia?, Nature Reviews Neurology 9, 211-221 (2013).

2. S. Fleminger, D. L. Oliver, S. Lovestone, S. Rabe-Hesketh, A. Giora, Head injury as a risk factor for Alzheimer's disease: the evidence 10 years on; a partial replication, Journal of Neurology, Neurosurgery \& Psychiatry 74, 857-862 (2003).

3. A. C. McKee, T. D. Stein, C. J. Nowinski, R. A. Stern, D. H. Daneshvar, V. E. Alvarez, H.-S. Lee, G. Hall, S. M. Wojtowicz, C. M. Baugh, D. O. Riley, C. A. Kubilus, K. A. Cormier, M. A. Jacobs, B. R. Martin, C. R. Abraham, T. Ikezu, R. R. Reichard, B. L. Wolozin, A. E. Budson, L. E. Goldstein, N. W. Kowall, R. C. Cantu, The spectrum of disease in chronic traumatic encephalopathy, Brain 136, 43-64 (2013).

4. K. Blennow, J. Hardy, H. Zetterberg, The neuropathology and neurobiology of traumatic brain injury, Neuron 76, 886-899 (2012).

5. J. a. N. Corsellis, C. J. Bruton, D. Freeman-Browne, The aftermath of boxing, Psychological Medicine 3, 270-303 (1973).

6. A. C. McKee, N. J. Cairns, D. W. Dickson, R. D. Folkerth, C. D. Keene, I. Litvan, D. P. Perl, T. D. Stein, J.-P. Vonsattel, W. Stewart, Y. Tripodis, J. F. Crary, K. F. Bieniek, K. Dams-O'Connor, V. E. Alvarez, W. A. Gordon, the T. Group, The first NINDS/NIBIB consensus meeting to define neuropathological criteria for the diagnosis of chronic traumatic encephalopathy, Acta Neuropathol 131, 75-86 (2016).

7. V. E. Johnson, W. Stewart, D. H. Smith, Widespread Tau and Amyloid-Beta Pathology Many Years After a Single Traumatic Brain Injury in Humans, Brain Pathology 22, 142-149 (2012).

8. H. T. Tran, F. M. LaFerla, D. M. Holtzman, D. L. Brody, Controlled cortical impact traumatic brain injury in 3xTg-AD mice causes acute intra-axonal amyloid- $\beta$ accumulation and independently accelerates the development of tau abnormalities, Journal of Neuroscience 31, 9513-9525 (2011).

9. H. T. Tran, L. Sanchez, D. L. Brody, Inhibition of JNK by a peptide inhibitor reduces traumatic brain injury-induced tauopathy in transgenic mice, Journal of Neuropathology \& Experimental Neurology 71, 116-129 (2012).

10. C.-F. Xia, J. Arteaga, G. Chen, U. Gangadharmath, L. F. Gomez, D. Kasi, C. Lam, Q. Liang, C. Liu, V. P. Mocharla, F. Mu, A. Sinha, H. Su, A. K. Szardenings, J. C. Walsh, E. Wang, C. Yu, W. Zhang, T. Zhao, H. C. Kolb, [18F]T807, a novel tau positron emission tomography imaging agent for Alzheimer's disease, Alzheimer's \& Dementia: The Journal of the Alzheimer's Association 9, 666-676 (2013). 
11. D. T. Chien, A. K. Szardenings, S. Bahri, J. C. Walsh, F. Mu, C. Xia, W. R. Shankle, A. J. Lerner, M.-Y. Su, A. Elizarov, Early clinical PET imaging results with the novel PHF-tau radioligand [F18]-T808, Journal of Alzheimer's Disease 38, 171-184 (2014).

12. M. Marquié, M. D. Normandin, C. R. Vanderburg, I. M. Costantino, E. A. Bien, L. G. Rycyna, W. E. Klunk, C. A. Mathis, M. D. Ikonomovic, M. L. Debnath, N. Vasdev, B. C. Dickerson, S. N. Gomperts, J. H. Growdon, K. A. Johnson, M. P. Frosch, B. T. Hyman, T. Gómez-Isla, Validating novel tau positron emission tomography tracer [F18]-AV-1451 (T807) on postmortem brain tissue, Ann Neurol. 78, 787-800 (2015).

13. K. Sander, T. Lashley, P. Gami, T. Gendron, M. F. Lythgoe, J. D. Rohrer, J. M. Schott, T. Revesz, N. C. Fox, E. \AArstad, Characterization of tau positron emission tomography tracer [18 F] AV-1451 binding to postmortem tissue in Alzheimer's disease, primary tauopathies, and other dementias, Alzheimer's \& Dementia 12, 1116-1124 (2016).

14. M. Marquié, M. D. Normandin, A. C. Meltzer, M. Siao Tick Chong, N. V. Andrea, A. Antón-Fernández, W. E. Klunk, C. A. Mathis, M. D. Ikonomovic, M. Debnath, others, Pathological correlations of [F-18]-AV-1451 imaging in non-alzheimer tauopathies, Annals of neurology 81, 117-128 (2017).

15. C. Vermeiren, P. Motte, D. Viot, G. Mairet-Coello, J.-P. Courade, M. Citron, J. Mercier, J. Hannestad, M. Gillard, The tau positron-emission tomography tracer AV1451 binds with similar affinities to tau fibrils and monoamine oxidases, Mov. Disord. 33, 273-281 (2018).

16. C. M. Lee, H. I. L. Jacobs, M. Marquié, J. A. Becker, N. V. Andrea, D. S. Jin, A. P. Schultz, M. P. Frosch, T. Gómez-Isla, R. A. Sperling, K. A. Johnson, 18F-Flortaucipir Binding in Choroid Plexus: Related to Race and Hippocampus Signal, J. Alzheimers Dis. 62, 1691-1702 (2018).

17. R. Ossenkoppele, D. R. Schonhaut, M. Schöll, S. N. Lockhart, N. Ayakta, S. L. Baker, J. P. O’Neil, M. Janabi, A. Lazaris, A. Cantwell, others, Tau PET patterns mirror clinical and neuroanatomical variability in Alzheimer's disease, Brain 139, 1551-1567 (2016).

18. D. Tosun, S. Landau, P. S. Aisen, R. C. Petersen, M. Mintun, W. Jagust, M. W. Weiner, A. D. N. Initiative, Association between tau deposition and antecedent amyloid$\beta$ accumulation rates in normal and early symptomatic individuals, Brain 140, 14991512 (2017).

19. L. Passamonti, P. Vázquez Rodríguez, Y. T. Hong, K. S. Allinson, D. Williamson, R. J. Borchert, S. Sami, T. E. Cope, W. R. Bevan-Jones, P. S. Jones, others, 18F-AV-1451 positron emission tomography in Alzheimer's disease and progressive supranuclear palsy, Brain 140, 781-791 (2017).

20. A. J. Schwarz, P. Yu, B. B. Miller, S. Shcherbinin, J. Dickson, M. Navitsky, A. D. Joshi, M. D. Devous Sr, M. S. Mintun, Regional profiles of the candidate tau PET ligand 
18 F-AV-1451 recapitulate key features of Braak histopathological stages, Brain 139, 1539-1550 (2016).

21. R. Smith, A. Puschmann, M. Schöll, T. Ohlsson, J. Van Swieten, M. Honer, E. Englund, O. Hansson, 18F-AV-1451 tau PET imaging correlates strongly with tau neuropathology in MAPT mutation carriers, Brain 139, 2372-2379 (2016).

22. C. Xia, S. J. Makaretz, C. Caso, S. McGinnis, S. N. Gomperts, J. Sepulcre, T. Gomez-Isla, B. T. Hyman, A. Schultz, N. Vasdev, others, Association of in vivo [18F] AV-1451 tau PET imaging results with cortical atrophy and symptoms in typical and atypical Alzheimer disease, Jama neurology 74, 427-436 (2017).

23. L. Wang, T. L. Benzinger, Y. Su, J. Christensen, K. Friedrichsen, P. Aldea, J. McConathy, N. J. Cairns, A. M. Fagan, J. C. Morris, others, Evaluation of tau imaging in staging Alzheimer disease and revealing interactions between $\beta$-amyloid and tauopathy, JAMA neurology 73, 1070-1077 (2016).

24. R. A. Stern, C. H. Adler, K. Chen, M. Navitsky, J. Luo, D. W. Dodick, M. L. Alosco, Y. Tripodis, D. D. Goradia, B. Martin, D. Mastroeni, N. G. Fritts, J. Jarnagin, M. D. Devous, M. A. Mintun, M. J. Pontecorvo, M. E. Shenton, E. M. Reiman, Tau PositronEmission Tomography in Former National Football League Players, New England Journal of Medicine 0, null (2019).

25. D. L. Dickstein, M. Y. Pullman, C. Fernandez, J. A. Short, L. Kostakoglu, K. Knesaurek, L. Soleimani, B. D. Jordan, W. A. Gordon, K. Dams-O’Connor, B. N. Delman, E. Wong, C. Y. Tang, S. T. DeKosky, J. R. Stone, R. C. Cantu, M. Sano, P. R. Hof, S. Gandy, Cerebral [18 F]T807/AV1451 retention pattern in clinically probable CTE resembles pathognomonic distribution of CTE tauopathy, Transl Psychiatry 6, e900 (2016).

26. G. M. Teasdale, G. D. Murray, J. a. R. Nicoll, The association between APOE $\varepsilon 4$, age and outcome after head injury: a prospective cohort study, Brain 128, 2556-2561 (2005).

27. K. Millar, J. A. R. Nicoll, S. Thornhill, G. D. Murray, G. M. Teasdale, Long term neuropsychological outcome after head injury: relation to APOE genotype, Journal of Neurology, Neurosurgery \& Psychiatry 74, 1047-1052 (2003).

28. D. J. Sharp, G. Scott, R. Leech, Network dysfunction after traumatic brain injury, Nature Reviews. Neurology 10, 156 (2014).

29. K. M. Kinnunen, R. Greenwood, J. H. Powell, R. Leech, P. C. Hawkins, V. Bonnelle, M. C. Patel, S. J. Counsell, D. J. Sharp, White matter damage and cognitive impairment after traumatic brain injury, Brain 134, 449-463 (2011).

30. V. E. Johnson, W. Stewart, D. H. Smith, Traumatic brain injury and amyloid- $\beta$ pathology: a link to Alzheimer's disease?, Nature Reviews Neuroscience 11, 361-370 (2010). 
31. D. H. Smith, V. E. Johnson, J. Q. Trojanowski, W. Stewart, Chronic traumatic encephalopathy — confusion and controversies, Nature Reviews Neurology 15, 179 (2019).

32. R. Harada, N. Okamura, S. Furumoto, T. Tago, K. Yanai, H. Arai, Y. Kudo, Characteristics of Tau and Its Ligands in PET Imaging, Biomolecules 6, 7 (2016).

33. K. Noda, K. Sasaki, K. Fujimi, Y. Wakisaka, Y. Tanizaki, Y. Wakugawa, Y. Kiyohara, M. Iida, H. Aizawa, T. Iwaki, Quantitative analysis of neurofibrillary pathology in a general population to reappraise neuropathological criteria for senile dementia of the neurofibrillary tangle type (tangle-only dementia): The Hisayama study, Neuropathology 26, 508-518 (2006).

34. E. D. Agdeppa, V. Kepe, J. Liu, S. Flores-Torres, N. Satyamurthy, A. Petric, G. M. Cole, G. W. Small, S.-C. Huang, J. R. Barrio, Binding Characteristics of Radiofluorinated 6-Dialkylamino-2-Naphthylethylidene Derivatives as Positron Emission Tomography Imaging Probes for $\beta$-Amyloid Plaques in Alzheimer's Disease, J. Neurosci. 21, RC189-RC189 (2001).

35. H. Braak, E. Braak, Staging of alzheimer's disease-related neurofibrillary changes, Neurobiology of Aging 16, 271-278 (1995).

36. O. Hansson, H. Zetterberg, P. Buchhave, E. Londos, K. Blennow, L. Minthon, Association between CSF biomarkers and incipient Alzheimer's disease in patients with mild cognitive impairment: a follow-up study, The Lancet Neurology 5, 228-234 (2006).

37. M. B. Aerts, R. a. J. Esselink, B. R. Bloem, M. M. Verbeek, Cerebrospinal fluid tau and phosphorylated tau protein are elevated in corticobasal syndrome, Mov. Disord. 26, 169-173 (2011).

38. N. Andreasen, L. Minthon, P. Davidsson, E. Vanmechelen, H. Vanderstichele, B. Winblad, K. Blennow, Evaluation of CSF-tau and CSF-A $\beta 42$ as diagnostic markers for Alzheimer disease in clinical practice, Archives of Neurology 58, 373-379 (2001).

39. H. Zetterberg, M. A. Hietala, M. Jonsson, N. Andreasen, E. Styrud, I. Karlsson, AAke Edman, C. Popa, A. Rasulzada, L.-O. Wahlund, Neurochemical aftermath of amateur boxing, Archives of neurology 63, 1277-1280 (2006).

40. G. Franz, R. Beer, A. Kampfl, K. Engelhardt, E. Schmutzhard, H. Ulmer, F. Deisenhammer, Amyloid beta 1-42 and tau in cerebrospinal fluid after severe traumatic brain injury, Neurology 60, 1457-1461 (2003).

41. L. Papa, L. M. Lewis, S. Silvestri, J. L. Falk, P. Giordano, G. M. Brophy, J. A. Demery, M. C. Liu, J. Mo, L. Akinyi, S. Mondello, K. Schmid, C. S. Robertson, F. C. Tortella, R. L. Hayes, K. K. W. Wang, Serum levels of Ubiquitin C-terminal Hydrolase (UCH-L1) distinguish mild traumatic brain injury (TBI) from trauma controls and are elevated in mild and moderate TBI patients with intracranial lesions and neurosurgical intervention, J Trauma Acute Care Surg 72, 1335-1344 (2012). 
42. K. D. Wilkinson, K. M. Lee, S. Deshpande, P. Duerksen-Hughes, J. M. Boss, J. Pohl, The neuron-specific protein PGP 9.5 is a ubiquitin carboxyl-terminal hydrolase, Science 246, 670-673 (1989).

43. K. Bilguvar, N. K. Tyagi, C. Ozkara, B. Tuysuz, M. Bakircioglu, M. Choi, S. Delil, A. O. Caglayan, J. F. Baranoski, O. Erturk, C. Yalcinkaya, M. Karacorlu, A. Dincer, M. H. Johnson, S. Mane, S. S. Chandra, A. Louvi, T. J. Boggon, R. P. Lifton, A. L. Horwich, M. Gunel, Recessive loss of function of the neuronal ubiquitin hydrolase UCHL1 leads to early-onset progressive neurodegeneration, PNAS 110, 3489-3494 (2013).

44. J. Choi, A. I. Levey, S. T. Weintraub, H. D. Rees, M. Gearing, L.-S. Chin, L. Li, Oxidative modifications and down-regulation of ubiquitin carboxyl-terminal hydrolase L1 associated with idiopathic Parkinson's and Alzheimer's diseases, J. Biol. Chem. 279, 13256-13264 (2004).

45. J. Lowe, H. McDermott, M. Landon, R. J. Mayer, K. D. Wilkinson, Ubiquitin carboxyl-terminal hydrolase (PGP 9.5) is selectively present in ubiquitinated inclusion bodies characteristic of human neurodegenerative diseases, The Journal of Pathology 161, 153-160 (1990).

46. A. Öhrfelt, P. Johansson, A. Wallin, U. Andreasson, H. Zetterberg, K. Blennow, J. Svensson, Increased Cerebrospinal Fluid Levels of Ubiquitin Carboxyl-Terminal Hydrolase L1 in Patients with Alzheimer's Disease, DEE 6, 283-294 (2016).

47. Y. Yoshiyama, K. Uryu, M. Higuchi, L. Longhi, R. Hoover, S. Fujimoto, T. McIntosh, V. M.-Y. Lee, J. Q. Trojanowski, Enhanced neurofibrillary tangle formation, cerebral atrophy, and cognitive deficits induced by repetitive mild brain injury in a transgenic tauopathy mouse model, Journal of neurotrauma 22, 1134-1141 (2005).

48. M. Ghajari, P. J. Hellyer, D. J. Sharp, Computational modelling of traumatic brain injury predicts the location of chronic traumatic encephalopathy pathology, Brain 140, 333-343 (2017).

49. C. S. Hill, M. P. Coleman, D. K. Menon, Traumatic Axonal Injury: Mechanisms and Translational Opportunities, Trends in Neurosciences 39, 311-324 (2016).

50. M. G. Spillantini, M. Goedert, Tau pathology and neurodegeneration, The Lancet Neurology 12, 609-622 (2013).

51. M. Polymenidou, D. W. Cleveland, Prion-like spread of protein aggregates in neurodegeneration, Journal of Experimental Medicine 209, 889-893 (2012).

52. G. Scott, A. F. Ramlackhansingh, P. Edison, P. Hellyer, J. Cole, M. Veronese, R. Leech, R. J. Greenwood, F. E. Turkheimer, S. M. Gentleman, R. A. Heckemann, P. M. Matthews, D. J. Brooks, D. J. Sharp, Amyloid pathology and axonal injury after brain trauma, Neurology 86, 821-828 (2016). 
53. S. Koponen, T. Taiminen, V. Kairisto, R. Portin, H. Isoniemi, S. Hinkka, O.

Tenovuo, APOE- $\varepsilon 4$ predicts dementia but not other psychiatric disorders after traumatic brain injury, Neurology 63, 749-750 (2004).

54. N. Nathoo, R. Chetty, J. R. van Dellen, G. H. Barnett, Genetic vulnerability following traumatic brain injury: the role of apolipoprotein E, Mol Pathol 56, 132-136 (2003).

55. Y. Shi, K. Yamada, S. A. Liddelow, S. T. Smith, L. Zhao, W. Luo, R. M. Tsai, S. Spina, L. T. Grinberg, J. C. Rojas, G. Gallardo, K. Wang, J. Roh, G. Robinson, M. B. Finn, H. Jiang, P. M. Sullivan, C. Baufeld, M. W. Wood, C. Sutphen, L. McCue, C. Xiong, J. L. Del-Aguila, J. C. Morris, C. Cruchaga, Alzheimer's Disease Neuroimaging Initiative, A. M. Fagan, B. L. Miller, A. L. Boxer, W. W. Seeley, O. Butovsky, B. A. Barres, S. M. Paul, D. M. Holtzman, ApoE4 markedly exacerbates tau-mediated neurodegeneration in a mouse model of tauopathy, Nature 549, 523-527 (2017).

56. L. Li, Y. Bao, S. He, G. Wang, Y. Guan, D. Ma, R. Wu, P. Wang, X. Huang, S. Tao, Q. Liu, Y. Wang, J. Yang, The Association Between Apolipoprotein E and Functional Outcome After Traumatic Brain Injury, Medicine (Baltimore) 94 (2015).

57. J. A. R. Nicoll, G. W. Roberts, D. I. Graham, Apolipoprotein E $\varepsilon 4$ allele is associated with deposition of amyloid $\beta$-protein following head injury, Nature Medicine 1, 135-137 (1995).

58. R. E. Hartman, H. Laurer, L. Longhi, K. R. Bales, S. M. Paul, T. K. McIntosh, D. M. Holtzman, Apolipoprotein E4 Influences Amyloid Deposition But Not Cell Loss after Traumatic Brain Injury in a Mouse Model of Alzheimer's Disease, J. Neurosci. 22, 10083-10087 (2002).

59. L. Ewing-Cobbs, M. Barnes, Linguistic outcomes following traumatic brain injury in children, Seminars in Pediatric Neurology 9, 209-217 (2002).

60. S. J. Makaretz, M. Quimby, J. Collins, N. Makris, S. McGinnis, A. Schultz, N. Vasdev, K. A. Johnson, B. C. Dickerson, Flortaucipir tau PET imaging in semantic variant primary progressive aphasia, J. Neurol. Neurosurg. Psychiatry 89, 1024-1031 (2018).

61. J. F. Malec, A. W. Brown, C. L. Leibson, J. T. Flaada, J. N. Mandrekar, N. N. Diehl, P. K. Perkins, The Mayo classification system for traumatic brain injury severity, Journal of neurotrauma 24, 1417-1424 (2007).

62. A. A. Lammertsma, S. P. Hume, Simplified Reference Tissue Model for PET Receptor Studies, NeuroImage 4, 153-158 (1996).

63. R. N. Gunn, A. A. Lammertsma, S. P. Hume, V. J. Cunningham, Parametric Imaging of Ligand-Receptor Binding in PET Using a Simplified Reference Region Model, NeuroImage 6, 279-287 (1997). 
64. J. Mez, D. H. Daneshvar, P. T. Kiernan, B. Abdolmohammadi, V. E. Alvarez, B. R. Huber, M. L. Alosco, T. M. Solomon, C. J. Nowinski, L. McHale, K. A. Cormier, C. A. Kubilus, B. M. Martin, L. Murphy, C. M. Baugh, P. H. Montenigro, C. E. Chaisson, Y. Tripodis, N. W. Kowall, J. Weuve, M. D. McClean, R. C. Cantu, L. E. Goldstein, D. I. Katz, R. A. Stern, T. D. Stein, A. C. McKee, Clinicopathological Evaluation of Chronic Traumatic Encephalopathy in Players of American Football, JAMA 318, 360-370 (2017).

65. R Core Team, others, R: A language and environment for statistical computing, (2014) (available at http://www.R-project.org/).

66. A. M. Winkler, G. R. Ridgway, M. A. Webster, S. M. Smith, T. E. Nichols, Permutation inference for the general linear model, NeuroImage 92, 381-397 (2014).

67. R. S. Desikan, F. Ségonne, B. Fischl, B. T. Quinn, B. C. Dickerson, D. Blacker, R. L. Buckner, A. M. Dale, R. P. Maguire, B. T. Hyman, An automated labeling system for subdividing the human cerebral cortex on MRI scans into gyral based regions of interest, Neuroimage 31, 968-980 (2006).

68. K. Hua, J. Zhang, S. Wakana, H. Jiang, X. Li, D. S. Reich, P. A. Calabresi, J. J. Pekar, P. C. van Zijl, S. Mori, Tract probability maps in stereotaxic spaces: analyses of white matter anatomy and tract-specific quantification, Neuroimage 39, 336-347 (2008).

69. A. F. Ramlackhansingh, D. J. Brooks, R. J. Greenwood, S. K. Bose, F. E. Turkheimer, K. M. Kinnunen, S. Gentleman, R. A. Heckemann, K. Gunanayagam, G. Gelosa, D. J. Sharp, Inflammation after trauma: Microglial activation and traumatic brain injury, Ann Neurol. 70, 374-383 (2011).

70. H. Zetterberg, K. Blennow, Fluid biomarkers for mild traumatic brain injury and related conditions, Nature Reviews Neurology 12, 563-574 (2016).

71. J. Ashburner, K. J. Friston, Unified segmentation, NeuroImage 26, 839-851 (2005).

72. J. Ashburner, A fast diffeomorphic image registration algorithm, NeuroImage 38, 95-113 (2007).

73. A. C. Tziortzi, G. E. Searle, S. Tzimopoulou, C. Salinas, J. D. Beaver, M. Jenkinson, M. Laruelle, E. A. Rabiner, R. N. Gunn, Imaging dopamine receptors in humans with [11C]-(+)-PHNO: Dissection of D3 signal and anatomy, NeuroImage 54, 264-277 (2011).

74. M. Jenkinson, P. Bannister, M. Brady, S. Smith, Improved Optimization for the Robust and Accurate Linear Registration and Motion Correction of Brain Images, NeuroImage 17, 825-841 (2002).

75. J. Ashburner, K. J. Friston, Voxel-Based Morphometry-The Methods, NeuroImage 11, 805-821 (2000). 
76. P. Ripollés, J. Marco-Pallarés, R. de Diego-Balaguer, J. Miró, M. Falip, M. Juncadella, F. Rubio, A. Rodriguez-Fornells, Analysis of automated methods for spatial normalization of lesioned brains, NeuroImage 60, 1296-1306 (2012).

77. J. M. S. Pereira, L. Xiong, J. Acosta-Cabronero, G. Pengas, G. B. Williams, P. J. Nestor, Registration accuracy for VBM studies varies according to region and degenerative disease grouping, NeuroImage 49, 2205-2215 (2010).

78. A. Mechelli, C. J. Price, K. J. Friston, J. Ashburner, Voxel-based morphometry of the human brain: methods and applications, Current medical imaging reviews 1, 105-113 (2005).

79. H. Zhang, B. B. Avants, P. A. Yushkevich, J. H. Woo, S. Wang, L. F. McCluskey, L. B. Elman, E. R. Melhem, J. C. Gee, High-Dimensional Spatial Normalization of Diffusion Tensor Images Improves the Detection of White Matter Differences: An Example Study Using Amyotrophic Lateral Sclerosis, IEEE Transactions on Medical Imaging 26, 1585-1597 (2007).

80. S. M. Smith, M. Jenkinson, H. Johansen-Berg, D. Rueckert, T. E. Nichols, C. E. Mackay, K. E. Watkins, O. Ciccarelli, M. Z. Cader, P. M. Matthews, others, Tract-based spatial statistics: voxelwise analysis of multi-subject diffusion data, Neuroimage 31, 1487-1505 (2006).

81. B. Fischl, FreeSurfer, Neuroimage 62, 774-781 (2012).

82. C. Destrieux, B. Fischl, A. Dale, E. Halgren, Automatic parcellation of human cortical gyri and sulci using standard anatomical nomenclature, Neuroimage 53, 1-15 (2010). 


\section{Acknowledgments:}

Funding: Project funded by the Medical Research Council (MRC) UK grant number: MR/L022141/1 (to DJS). DJS was supported by an NIHR Research Professorship (NIHR-RP-011-048). This work was supported by the UK Dementia Research Institute. Infrastructure supported by the National Institute for Health Research (NIHR) Biomedical Research Centre (BRC).

Author contributions: NG: acquisition, analysis and interpretation of data, drafting and critical revision of the article, final approval of the version to be published; LML: acquisition, analysis and interpretation of data, critical revision of the article, final approval of the version to be published; AW: analysis and interpretation of data, drafting and critical revision of the article, final approval of the version to be published; KAZ: acquisition of data, critical revision of the article, final approval of the version to be published; LM: acquisition of data, critical revision of the article, final approval of the version to be published; CML: acquisition of data, critical revision of the article, final approval of the version to be published; ER: acquisition of data, critical revision of the article, final approval of the version to be published; AH: analysis of data, critical revision of the article, final approval of the version to be published; HZ: design and interpretation of data, critical revision of the article, final approval of the version to be published; JP: design and interpretation of data, critical revision of the article, final approval of the version to be published; PMM: study design, interpretation of data, critical revision of the article, final approval of the version to be published; RNG: study design, interpretation of data, critical revision of the article, final approval of the version to be published; TMM: study design, interpretation of data, critical revision of the article, final approval of the version to be published; DJS: conception and design of the study, interpretation of data, drafting and critical revision of the article, final approval of the version to be published. 
Competing interests: None of the authors declare any competing interests related to the current study. HZ has served at scientific advisory boards for Roche Diagnostics, Wave, Samumed and $\operatorname{CogRx}$, has lectured for Alzecure, and is a co-founder of Brain Biomarker Solutions in Gothenburg AB, a GU Ventures-based platform company at the University of Gothenburg. PMM acknowledges generous personal and research support from the Edmond J Safra and Lily Safra Foundation, an NIHR Senior Investigator Award and the UK Dementia Research Institute. He is reimbursed for service on a Scientific Advisory Board to Ipsen Pharmaceuticals. He has received consultancy fees from Roche, Adelphi Communications, Celgene and Biogen OrbiMed. He has received honoraria or speakers' fees from Novartis and Biogen and has received research or educational funds from Biogen, Novartis and GlaxoSmithKline. RNG is a consultant for Abbvie, Biogen and Cerveau. NG, LML, AW, KAZ, LM, CML, ER, AH, JP, TMM and DJS have no disclosures.

Data availability: All the data are present in the main text or supplementary material 


\section{Tables}

Table 1

\begin{tabular}{|c|c|c|c|c|c|c|c|}
\hline Patient & Age & Sex & $\begin{array}{l}\text { TBI } \\
\text { etiology }\end{array}$ & $\begin{array}{l}\text { Time since } \\
\text { TBI (years) }\end{array}$ & $\begin{array}{l}\text { GOS-E } \\
\text { current } \\
\text { (change) }\end{array}$ & $\begin{array}{l}\text { MMSE } \\
\text { current } \\
\text { (change) }\end{array}$ & APOE \\
\hline $\mathrm{P} 1$ & 56 & Female & RTA & 35 & $5(0)$ & $22(-2)$ & $\varepsilon 3 / \varepsilon 3$ \\
\hline $\mathrm{P} 2$ & 39 & Female & RTA & 28 & $5(-1)$ & $27(-2)$ & $\varepsilon 3 / \varepsilon 3$ \\
\hline P3 & 61 & Female & RTA & 35 & $5(-1)$ & $28(-2)$ & $\varepsilon 3 / \varepsilon 3$ \\
\hline $\mathrm{P} 4$ & 39 & Male & RTA & 32 & $4(0)$ & $28(-1)$ & $\varepsilon 3 / \varepsilon 4$ \\
\hline P5 & 57 & Male & RTA & 35 & $8(0)$ & $30(0)$ & $\varepsilon 3 / \varepsilon 4$ \\
\hline P6 & 54 & Female & RTA & 36 & $5(-3)$ & $27(2)$ & $\varepsilon 3 / \varepsilon 4$ \\
\hline P7 & 65 & Male & Fall & 31 & $7(0)$ & $30(2)$ & $\varepsilon 3 / \varepsilon 4$ \\
\hline P8 & 29 & Female & RTA & 18 & 7 (NA) & 29 (NA) & $\varepsilon 3 / \varepsilon 3$ \\
\hline P9 & 49 & Male & RTA & 27 & 5 (NA) & 28 (NA) & $\varepsilon 2 / \varepsilon 3$ \\
\hline P10 & 48 & Male & RTA & 37 & $6(-2)$ & $29(-1)$ & $\varepsilon 3 / \varepsilon 3$ \\
\hline P11 & 46 & Female & RTA & 31 & $8(0)$ & $29(0)$ & $\varepsilon 3 / \varepsilon 3$ \\
\hline P12 & 44 & Male & RTA & 29 & $6(-1)$ & $24(-2)$ & $\varepsilon 3 / \varepsilon 3$ \\
\hline P13 & 54 & Male & RTA & 32 & $7(0)$ & 29 (NA) & $\varepsilon 3 / \varepsilon 3$ \\
\hline P14 & 57 & Male & RTA & 37 & $8(0)$ & $30(3)$ & $\varepsilon 3 / \varepsilon 3$ \\
\hline P15 & 54 & Male & RTA & 34 & $5(-1)$ & $29(2)$ & $\varepsilon 3 / \varepsilon 4$ \\
\hline P16 & 38 & Male & RTA & 19 & 8 (NA) & 29 (NA) & $\varepsilon 3 / \varepsilon 3$ \\
\hline P17 & 72 & Male & Assault & 51 & 5 (NA) & 27 (NA) & $\varepsilon 3 / \varepsilon 3$ \\
\hline P18 & 40 & Female & RTA & 31 & $5(0)$ & $23(-6)$ & $\varepsilon 3 / \varepsilon 4$ \\
\hline P19 & 43 & Male & RTA & 35 & $8(0)$ & $30(0)$ & $\varepsilon 3 / \varepsilon 3$ \\
\hline P20 & 55 & Male & Assault & 20 & 8 (NA) & 30 (NA) & $\varepsilon 3 / \varepsilon 4$ \\
\hline P21 & 43 & Male & RTA & 20 & 5 (NA) & 26 (NA) & $\varepsilon 4 / \varepsilon 4$ \\
\hline
\end{tabular}

Abbreviations: $A P O E$ : apolipoprotein E genotype; GOS-E = Glasgow Outcome Scale Extended; MMSE = Mini-Mental State Examination; NA = not available; RTA = road traffic accident; TBI = traumatic brain injury.

Table 1: Clinical characteristics of individual TBI participants. Participant designations correspond to those in Figures 1, 2 and S1. 
Table 2

\begin{tabular}{|c|c|c|c|c|c|c|c|c|c|}
\hline & \multirow[t]{2}{*}{ Outcome measure } & \multicolumn{4}{|c|}{ TBI vs. Healthy participants } & \multicolumn{4}{|c|}{ Disabled vs. Good recovery TBI subgroups } \\
\hline & & $\begin{array}{c}\text { TBI } \\
\text { mean }(+/-\mathrm{SD})\end{array}$ & $\begin{array}{c}\text { Controls } \\
\text { mean (+/- SD) }\end{array}$ & $\mathrm{W}$ & $P$ & $\begin{array}{l}\begin{array}{c}\text { Disabled TBI } \\
\text { subgroup }\end{array} \\
\text { mean }(+/-\mathrm{SD}) \\
\end{array}$ & $\begin{array}{c}\text { Good recovery TBI } \\
\text { subgroup } \\
\text { mean }(+/-\mathrm{SD}) \\
\end{array}$ & $\mathrm{W}$ & $\mathrm{P}$ \\
\hline \multicolumn{2}{|l|}{ Age (years) } & $49.7(10.3)$ & $54.2(12.1)$ & 80 & 0.16 & $49.9(10)$ & $49.3(11.2)$ & 35 & 0.29 \\
\hline \multicolumn{2}{|l|}{ Time since injury (years) } & $31.1(7.6)$ & - & - & - & $32.9(7.4)$ & $28.7(7.5)$ & 66.5 & 0.39 \\
\hline \multicolumn{2}{|l|}{ Education (years) } & $12.5(3.6)$ & $11.5(2.8)$ & 124.5 & 0.73 & $11.5(3.2)$ & $13.9(3.8)$ & 33.5 & 0.15 \\
\hline Premorbid intelligence & WTAR (scaled) & $101.4(16.4)$ & $110.3(10.8)$ & 76.5 & 0.13 & $92.6(16.1)$ & $113.1(6.8)$ & 9.5 & 0.002 \\
\hline \multirow[t]{3}{*}{ Cognitive impairment } & MMSE (current) & $27.8(2.3)$ & $29.5(0.9)$ & 57.5 & 0.017 & $26.5(2.3)$ & $29.6(0.5)$ & 4 & $<0.001$ \\
\hline & MMSE (change) & $-0.5(2.3)$ & - & - & - & $-1.3(2.4)$ & $1(1.4)$ & 7 & 0.041 \\
\hline & MMSE (change per year) & $-0.026(0.131)$ & - & - & - & $-0.073(0.134)$ & $0.058(0.081)$ & 7 & 0.045 \\
\hline Logical reasoning & WASI Matrix Reasoning & $22.7(8.2)$ & $25.2(7)$ & 87 & 0.46 & $18.1(7.3)$ & $28.9(4.4)$ & 8.5 & 0.001 \\
\hline \multirow[t]{4}{*}{ Processing speed } & Trail Making Test A (s) & $39.9(20.6)$ & $28.9(14.4)$ & 153 & 0.079 & $49.7(22.9)$ & $27.9(7.6)$ & 81 & 0.016 \\
\hline & Trail Making Test B (s) & $106.8(69.8)$ & $57.4(18.3)$ & 158 & 0.049 & $140.8(75.3)$ & $65.2(30.6)$ & 81 & 0.016 \\
\hline & Stroop Combined Baseline (s) & $50.8(10.8)$ & $41.1(8.5)$ & 175 & 0.017 & $52.7(12.3)$ & $48.2(8.6)$ & 67 & 0.38 \\
\hline & CRT RT (ms) & $579(121)$ & $512(103)$ & 160.5 & 0.077 & $645(115)$ & $491(53)$ & 94 & 0.003 \\
\hline \multirow[t]{4}{*}{ Executive function } & Trail Making Test B-A (s) & $66.9(56)$ & $28.5(8.7)$ & 145 & 0.15 & $91.1(63)$ & $37.3(26.3)$ & 79 & 0.028 \\
\hline & Stroop Inhibition-Switch(s) & $93(48.7)$ & $65.3(20.4)$ & 164 & 0.056 & $116.4(52.7)$ & $61.9(14.2)$ & 100 & $<0.001$ \\
\hline & $\begin{array}{c}\text { Stroop Inhibition-Switch vs. } \\
\text { Baseline (s) }\end{array}$ & $71.4(50.7)$ & $51.8(18.9)$ & 145 & 0.25 & $94.6(54.5)$ & $40.42(21.2)$ & 96 & 0.002 \\
\hline & FrSBe-Executive; current & $42.2(8.2)$ & $26.3(5.7)$ & 181.5 & $<0.001$ & $46(8.2)$ & $37.1(4.9)$ & 89.5 & 0.012 \\
\hline
\end{tabular}




\begin{tabular}{|c|c|c|c|c|c|c|c|c|c|}
\hline \multirow[t]{2}{*}{ Apathy } & LARS & $-21.5(10.1)$ & $-27.2(6.3)$ & 134 & 0.14 & $-17.2(10.7)$ & $-27.9(4.5)$ & 82 & 0.009 \\
\hline & FrSBe-Apathy; current & $31.9(9.6)$ & $21(5.1)$ & 157.5 & 0.005 & $34.3(10.5)$ & $28.8(7.8)$ & 71 & 0.24 \\
\hline \multirow[t]{2}{*}{ Impulsivity / Disinhibition } & BIS & $66.6(15.8)$ & $58.1(6.9)$ & 118.5 & 0.29 & $72.9(15)$ & $57.9(13.2)$ & 75 & 0.039 \\
\hline & FrSBe-Disinhibition; current & $33.5(10.3)$ & $24.3(5.3)$ & 143.5 & 0.028 & $36.1(11.5)$ & $30.1(7.7)$ & 70.5 & 0.25 \\
\hline \multirow[t]{5}{*}{ Verbal memory } & PT Immediate Recall & $18.3(8.7)$ & $19.8(8.2)$ & 102 & 0.61 & $16.4(9.2)$ & $20.4(7.8)$ & 39.5 & 0.32 \\
\hline & PT Delayed Recall & $6.4(3.8)$ & $7.3(3.6)$ & 100.5 & 0.56 & $5.6(3.9)$ & $7.6(3.5)$ & 38.5 & 0.28 \\
\hline & HVLT Immediate Recall & $21.2(5.3)$ & $25.5(4.7)$ & 63 & 0.038 & $19.4(4.9)$ & $23.7(5)$ & 25.5 & 0.046 \\
\hline & HVLT Delayed Recall & $6.3(3.6)$ & $8.8(2.5)$ & 65.5 & 0.048 & $5.3(3.9)$ & $7.7(2.7)$ & 34 & 0.16 \\
\hline & HVLT RDI & $9.2(2.4)$ & $9.9(2)$ & 97 & 0.47 & $8.7(2.8)$ & $10(1.5)$ & 40 & 0.33 \\
\hline \multirow[t]{4}{*}{ Visual memory } & $\begin{array}{l}\text { Paired Associates Learning (z- } \\
\text { score) }\end{array}$ & $-1.47(0.81)$ & $-0.73(0.92)$ & 66 & 0.043 & $-1.79(0.77)$ & $-1.03(0.69)$ & 25.5 & 0.036 \\
\hline & BVMT Immediate Recall & $16.2(8.3)$ & $21.7(5.5)$ & 63 & 0.039 & $10.9(3.7)$ & $23.3(7.5)$ & 7 & $<0.001$ \\
\hline & BVMT Delayed Recall & $6.8(3.7)$ & $9.5(1.4)$ & 64.5 & 0.044 & $4.7(3.1)$ & $9.6(2.4)$ & 12 & 0.003 \\
\hline & BVMT RDI & $4.9(1.3)$ & $5.9(0.3)$ & 67.5 & 0.026 & $4.3(1.4)$ & $5.9(0.3)$ & 16 & 0.004 \\
\hline Visuospatial ability & Feature match test (z-score) & $-1.46(0.74)$ & $-1.19(0.92)$ & 94.5 & 0.42 & $-1.88(0.69)$ & $-0.89(0.31)$ & 6 & $<0.001$ \\
\hline \multirow[t]{2}{*}{ Mood disturbance } & HADS-Anxiety & $8(6)$ & $3.9(3.8)$ & 161.5 & 0.069 & $9.9(5.6)$ & $5.3(5.7)$ & 80.5 & 0.063 \\
\hline & HADS-Depression & $4.5(4.7)$ & $1.9(2.5)$ & 156 & 0.11 & $6.4(5.1)$ & $1.8(2.4)$ & 86.5 & 0.022 \\
\hline
\end{tabular}

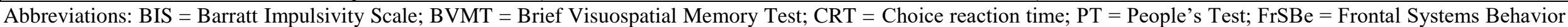

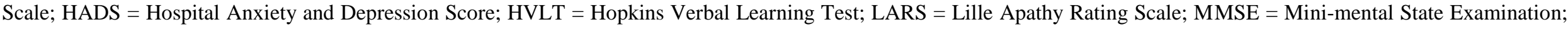

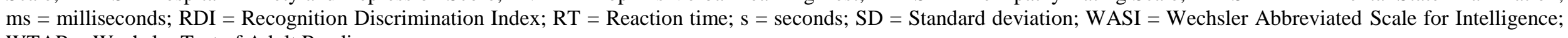
WTAR $=$ Wechsler Test of Adult Reading.

\section{Table 2: Demographic and neuropsychological comparisons between TBI and healthy control groups, as well as between disabled}

TBI and good recovery TBI subgroups. Independent sample Mann-Whitney-Wilcoxon test $\mathrm{W}$ and $\mathrm{P}$-values (P<0.05 in bold) for comparisons between groups and subgroups. 


\section{Figures}

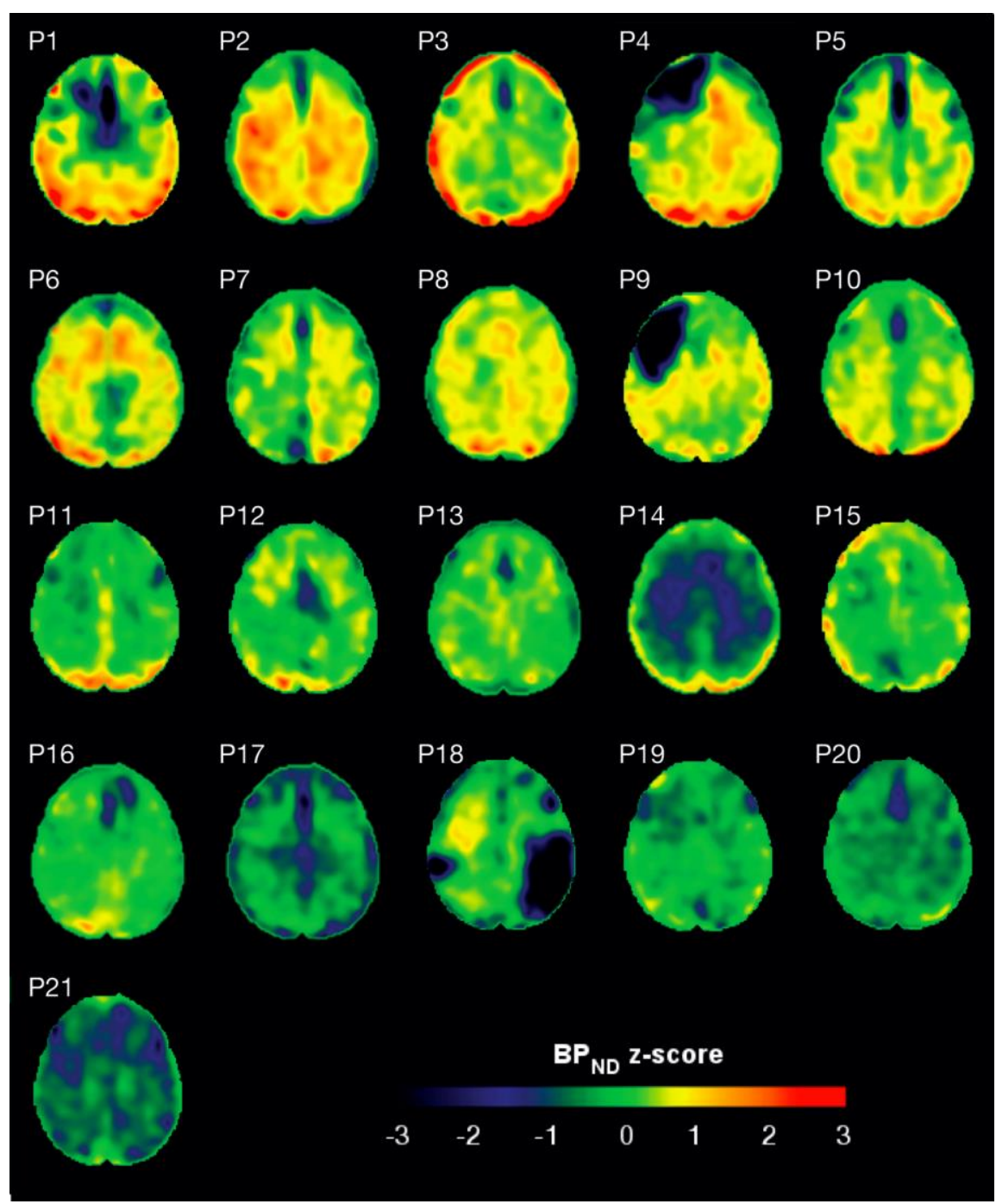

Fig. 1: Flortaucipir BPND z-score maps for each TBI individual are compared voxelwise to healthy controls. TBI participants are presented in descending order of number of voxels with flortaucipir $\mathrm{BP}_{\mathrm{ND}} \mathrm{z}>1.645$ (see Fig. 2). Axial images are displayed in radiological convention at MNI coordinate 55. Patchy cortical and subcortical increase in tracer uptake is observed in some TBI participants, most consistently in the lateral occipital cortex (P1 to P8 and P10), whereas others show similar $\mathrm{BP}_{\mathrm{ND}}$ values as controls (P17 to P21). The corresponding clinical characteristics are presented in Table 1. Participant designations correspond to those in Table 1, Figure 2 and fig. S1. 


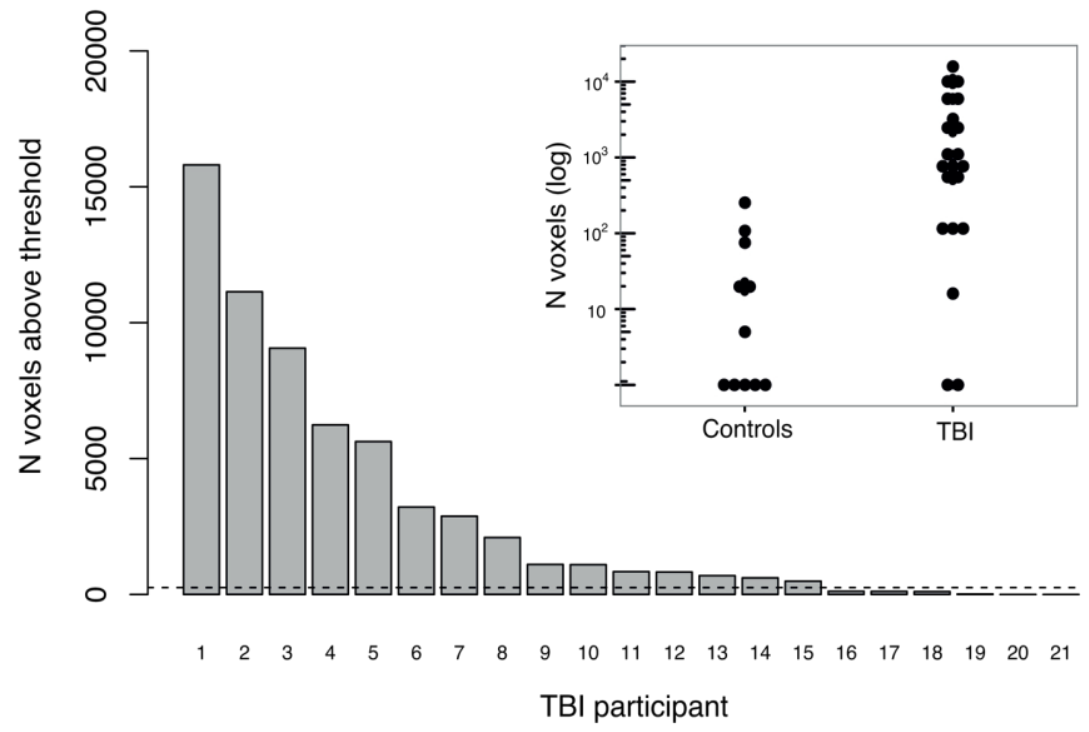

Fig. 2: The spatial extent of increased flortaucipir binding in TBI is expressed as the number of voxels with flortaucipir BPND $\mathrm{z}>1.645$ for each TBI patient compared to the healthy control group. The dotted line represents the number of voxels above threshold in the healthy control subject with the maximum number of voxels above that threshold (compared to the rest of the controls). Participant designations correspond to those in Table 1, Figure 1 and fig. S1. The inset figure shows the number of voxels above threshold (in logarithmic scale) in TBI and healthy controls. 


\section{Flortaucipir $\mathrm{BP}_{\mathrm{ND}} \mathrm{TBI}>$ healthy controls}

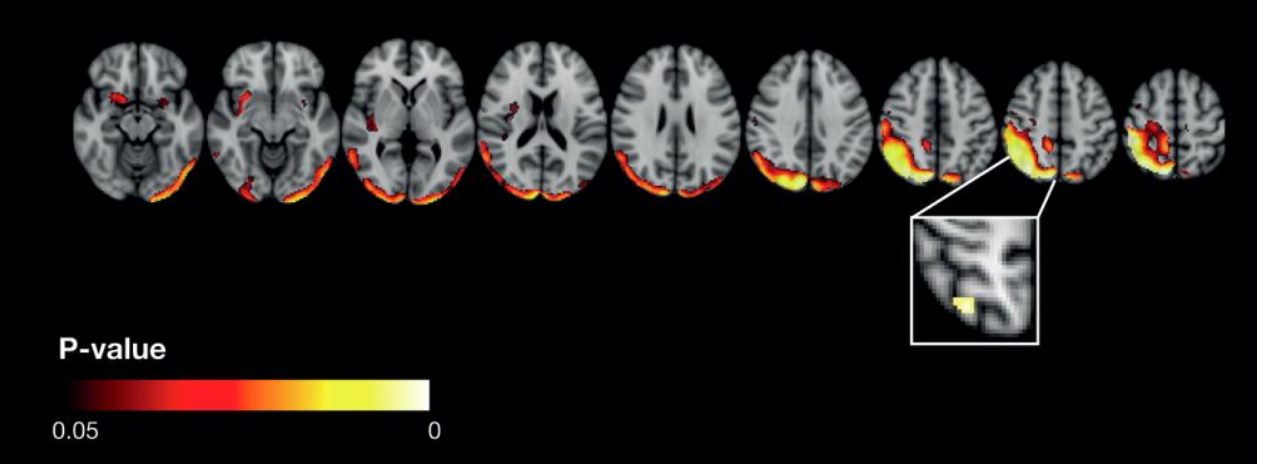

Fig. 3: Flortaucipir BPND is increased in TBI compared to healthy controls. Voxels with increased flortaucipir uptake in TBI at $\mathrm{P}<0.05$, not adjusted for multiple comparisons, are shown on axial slices for illustration. Flortaucipir binding was increased in TBI in a small cluster of voxels in the right parietal cortex (inset, $\mathrm{P}<0.05$, corrected for multiple comparisons).
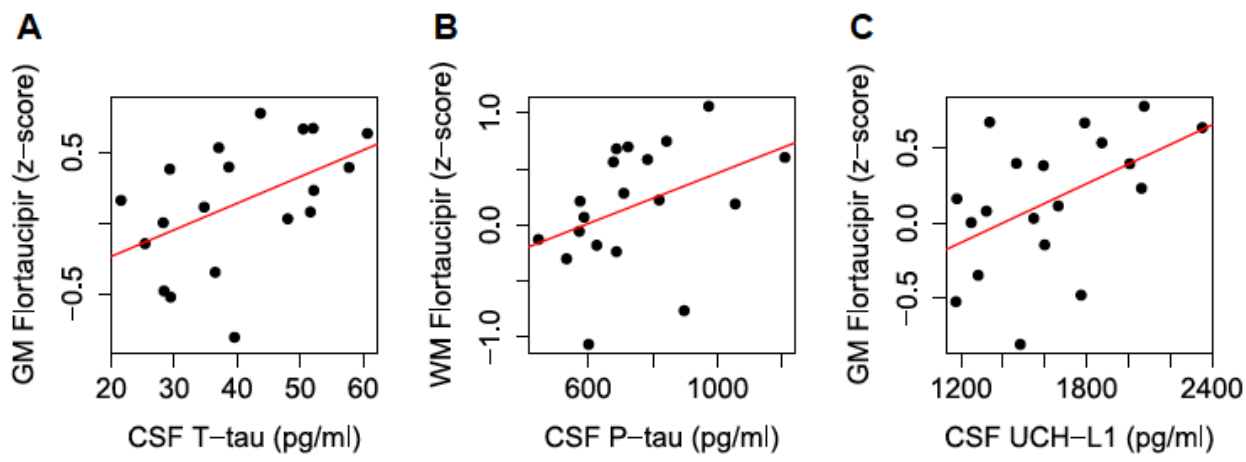

Fig. 4: Flortaucipir binding is associated with CSF biomarkers in TBI. CSF total tau (T-tau, A), phosphorylated (P-tau, B) and UCH-L1 (C) concentrations in the CSF are positively correlated with flortaucipir $\mathrm{BP}_{\mathrm{ND}}$ (normalized against the healthy control group) in the cerebral grey matter (T-tau, UCH-L1) and in the cerebral white matter (P-tau) in TBI participants. 
A

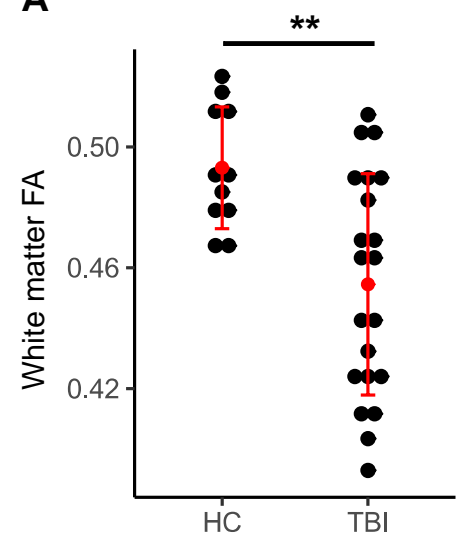

D

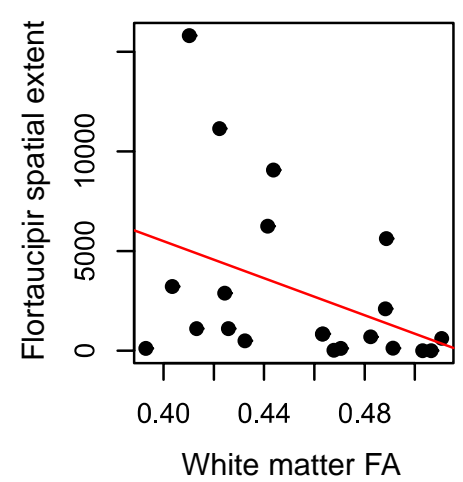

B

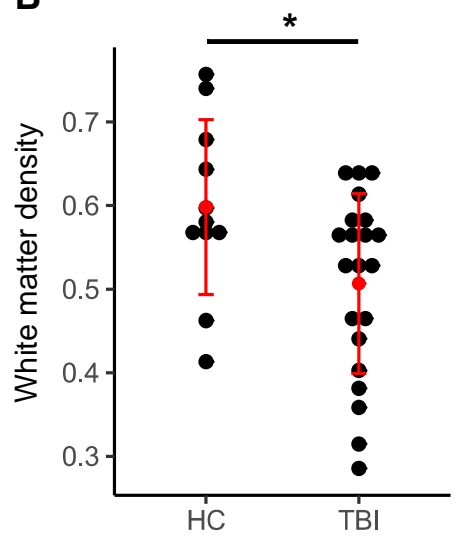

E

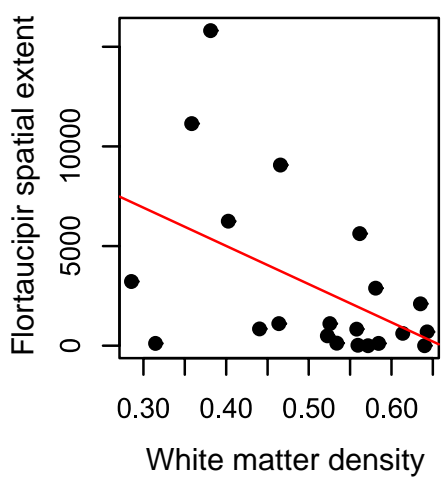

C

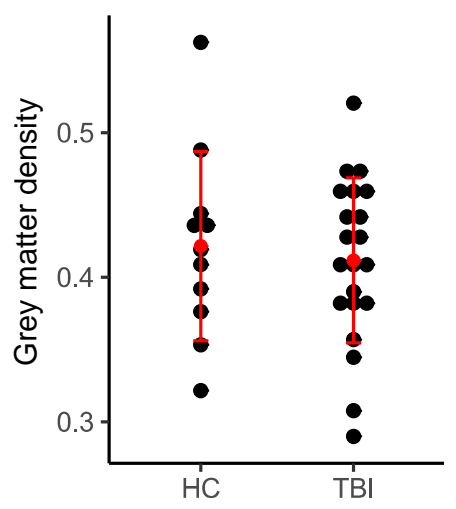

$\mathbf{F}$

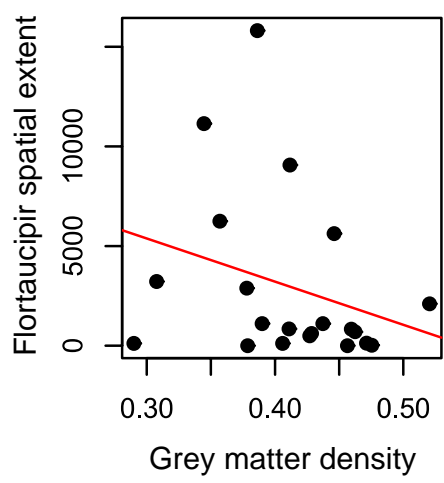

Fig. 5: White matter microstructural changes in TBI are associated with flortaucipir spatial extent. A) Cerebral white matter fractional anisotropy (FA) and B) VBM-derived white matter tissue density are reduced in TBI when compared to healthy controls (HC). This was not observed with grey matter tissue density (C). Flortaucipir spatial extent (number of voxels above threshold) in TBI is associated with reduced white matter FA (D) and white matter tissue density $(\mathbf{E})$, but not with grey matter tissue density $(\mathbf{F}) .{ }^{*} * \mathrm{P}<0.005$, $* \mathrm{P}<0.05$. 


\section{Fractional anisotropy}

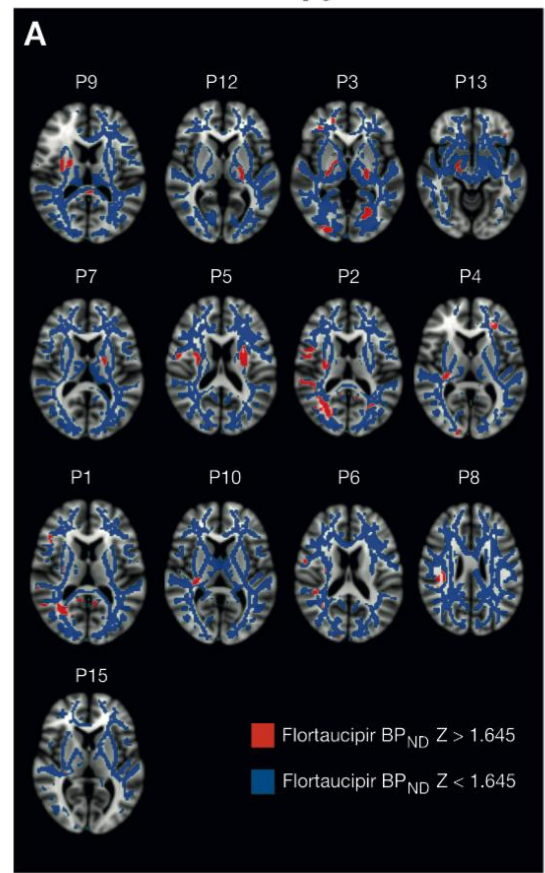

\section{White matter density}

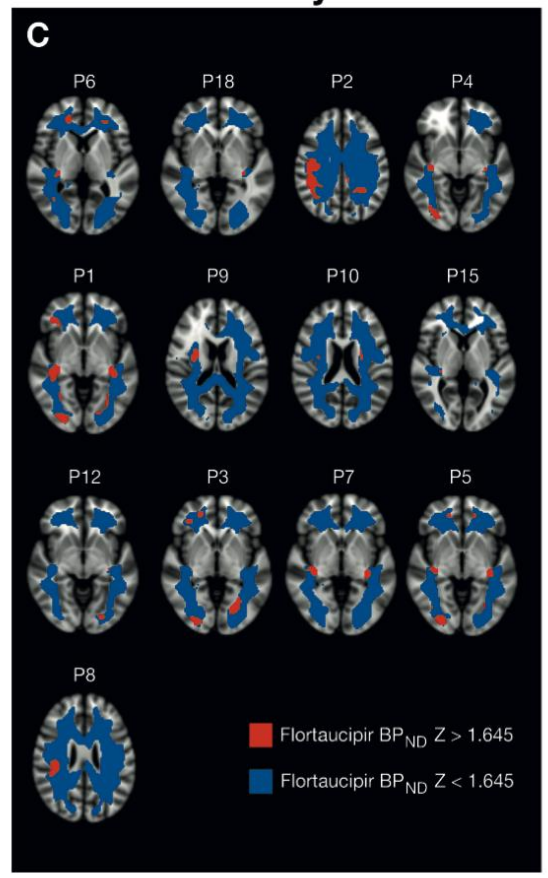

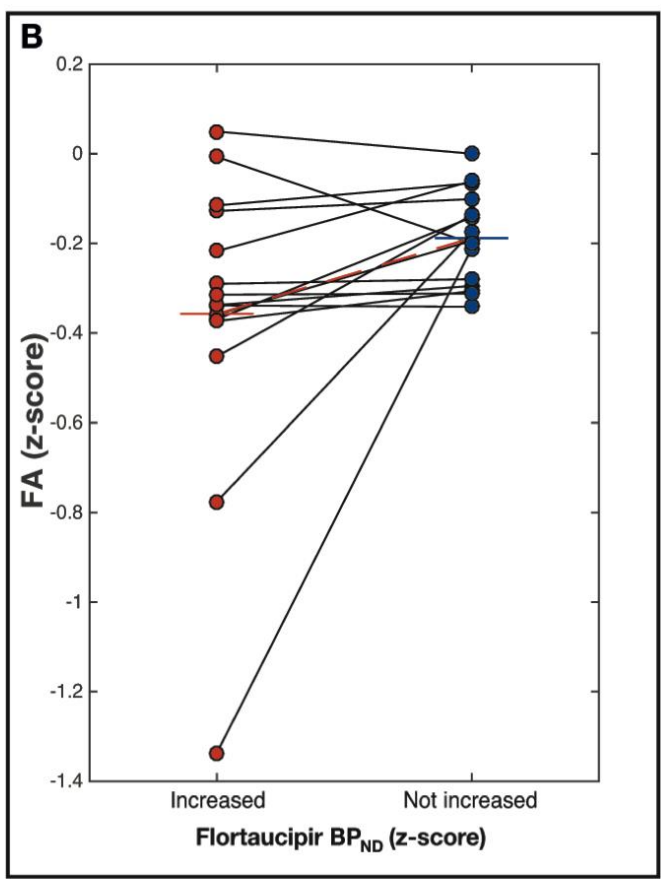

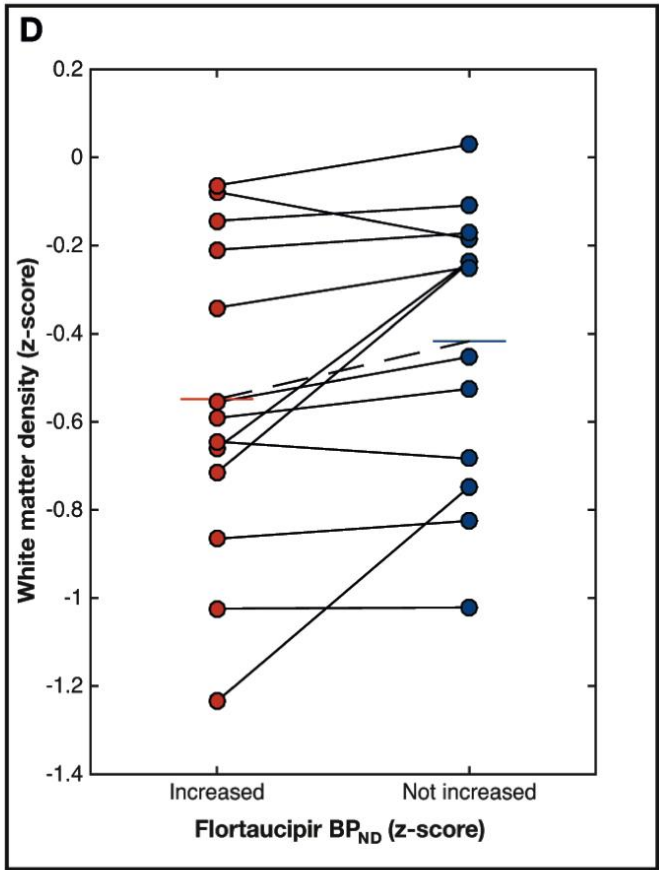

Fig. 6: Increased white matter flortaucipir $B P_{N D}$ co-localizes with white matter microstructural changes in TBI. Skeletonized FA (A) and white matter density (C), standardized against controls, were compared within subject between areas of increased 
flortaucipir binding (in red) and areas where binding was not increased (in blue). A representative axial slice for each patient shows the spatial distribution of the white matter areas compared and images are presented in order of greatest to smallest effect size (greatest decrease in FA or white matter density z-score in high flortaucipir areas shown top left). Both FA z-scores (B) and white matter density z-scores (D) are lower within areas of increased flortaucipir binding, defined as $\mathrm{BP}_{\mathrm{ND}} \mathrm{Z}>1.645$. 


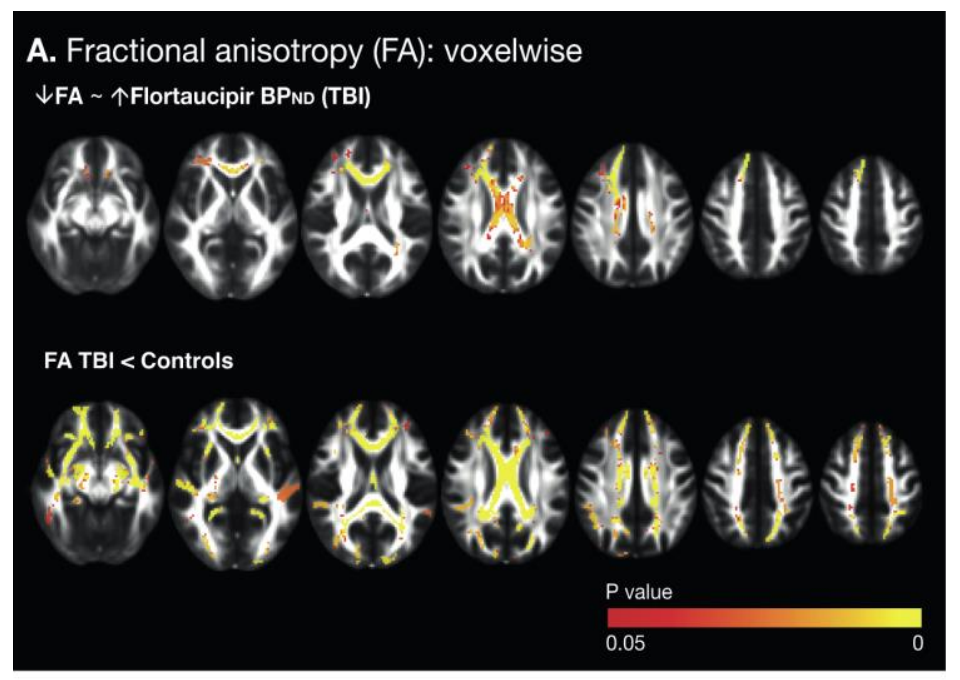

B. Fractional anisotropy $(\mathrm{FA})$ : tracts
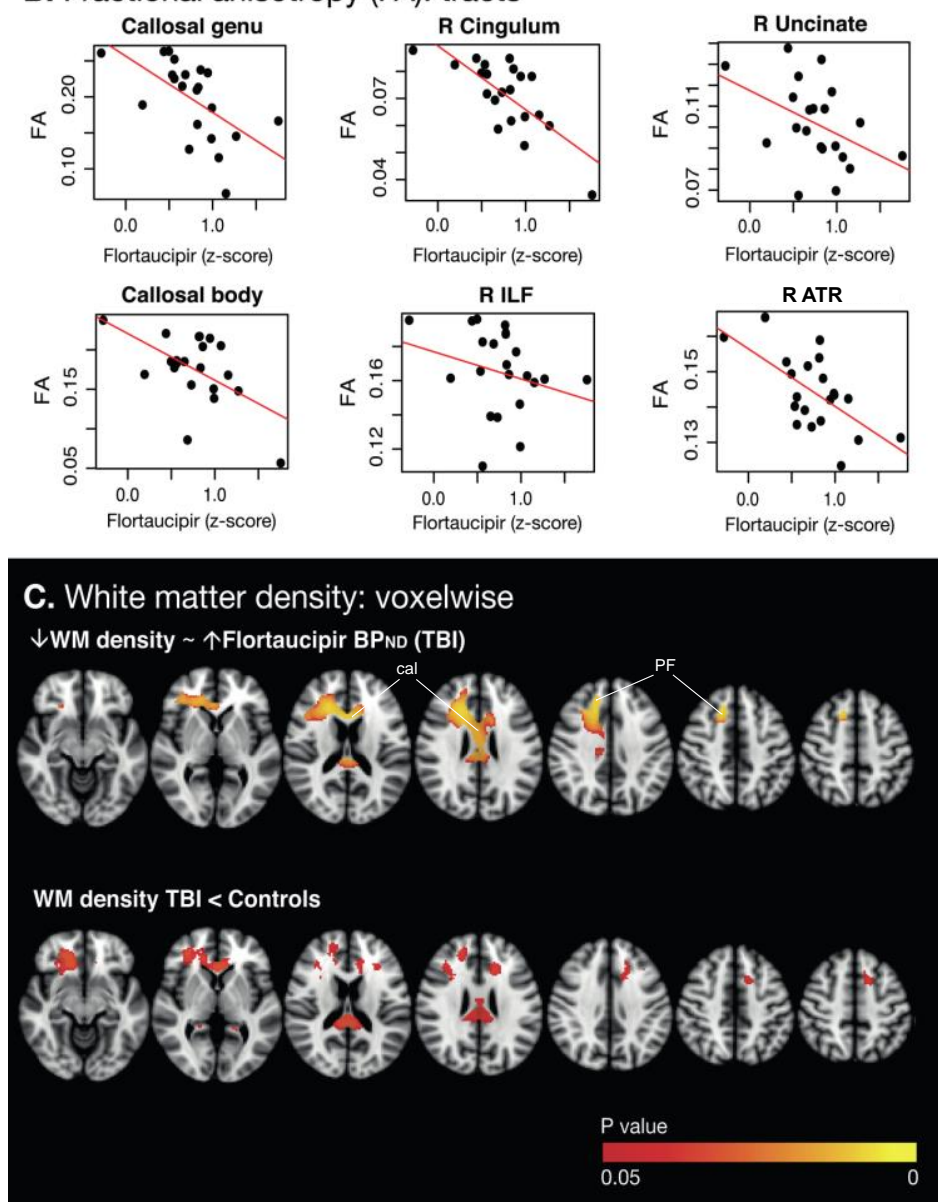

Fig. 7: Increased cortical flortaucipir binding in the right lateral occipital area is associated with microstructural white matter damage in TBI. A) Increased flortaucipir $\mathrm{BP}_{\mathrm{ND}}$ in the right lateral occipital cortex in TBI participants was associated with reduced 
diffusion MRI-derived fractional anisotropy (FA) in remote white matter tracts (upper panel), partly overlapping with white matter tracts of reduced FA in TBI when compared to controls (lower panel). B) Flortaucipir $\mathrm{BP}_{\mathrm{ND}}$ in the right lateral occipital cortex in $\mathrm{TBI}$ participants correlated negatively with average skeletonized FA within the genu and body of the corpus callosum as well as within the cingulum bundle, inferior longitudinal fasciculus (ILF), anterior thalamic radiation (ATR) and uncinate fasciculus in the right hemisphere. Correlation coefficients and P-values presented in Table S2. C) Upper panel: increased flortaucipir $\mathrm{BP}_{\mathrm{ND}}$ in the right lateral occipital cortex in $\mathrm{TBI}$ participants is associated with reduced voxel-based morphometry-derived white matter density in the corpus callosum (cal) and right prefrontal area (PF). This distribution partly overlaps with areas of white matter atrophy in TBI when compared to controls (lower panel). Results presented in radiological convention, color maps represent $\mathrm{P}$ values $<0.05$ adjusted for multiple comparisons. 